University of Wollongong

Research Online

Faculty of Engineering and Information

Faculty of Engineering and Information

Sciences - Papers: Part B

Sciences

2018

\title{
Lattice parameter evolution during heating of Ti-45Al-7.5Nb-0.25/0.5C alloys under atmospheric and high pressures
}

\author{
$\mathrm{Xi} \mathrm{Li}$ \\ University of Wollongong, Australian Nuclear Science and Technology Organisation, \\ x1910@uowmail.edu.au \\ Rian J. Dippenaar \\ University of Wollongong, rian@uow.edu.au \\ Ayumi Shiro \\ National Institute for Quantum and Radiological Science and Technology \\ Takahisa Shobu \\ Japan Atomic Energy Agency \\ Yuji Higo \\ Japan Synchrotron Radiation Research Institute
}

See next page for additional authors

Follow this and additional works at: https://ro.uow.edu.au/eispapers1

Part of the Engineering Commons, and the Science and Technology Studies Commons

Research Online is the open access institutional repository for the University of Wollongong. For further information contact the UOW Library: research-pubs@uow.edu.au 


\title{
Lattice parameter evolution during heating of Ti-45Al-7.5Nb-0.25/0.5C alloys under atmospheric and high pressures
}

\author{
Abstract \\ Lattice strain evolution during deformation processing of Ti-Al alloys at high temperature is important in \\ terms of its microstructural evolution and microstructural stability. It is shown here that careful evaluation \\ of lattice parameters is critical for the understanding of thermal expansion, crystallographic order, \\ chemical composition and response to pressure, allowing to identify phase transitions and segregation, in \\ addition to the measurement of the more conventional quantities phase composition and order \\ parameter. The lattice parameters of Ti-45Al-7.5Nb-0.25/0.5C (at. \%) alloys were calculated using both \\ Rietveld and single peak fitting methods from data obtained by in-situ synchrotron diffraction experiments \\ at high temperature under atmospheric and high pressure respectively. The lattice strain evolution as a \\ function of temperature in a Ti-45Al-7.5Nb-0.25C (at. \%) alloy under high pressure was compared with \\ that of a Ti-45Al-7.5Nb-0.5C (at. \%) alloy heated under atmospheric pressure. The contribution of each of \\ the four lattice strain factors is semi-quantitatively assessed in the temperature range investigated.

\section{Disciplines} \\ Engineering | Science and Technology Studies

\section{Publication Details} \\ Li, X., Dippenaar, R., Shiro, A., Shobu, T., Higo, Y., Reid, M., Suzuki, H., Akita, K., Funakoshi, K. \& Liss, K. \\ (2018). Lattice parameter evolution during heating of Ti-45Al-7.5Nb-0.25/0.5C alloys under atmospheric \\ and high pressures. Intermetallics, 102 120-131. \\ Authors \\ Xi Li, Rian J. Dippenaar, Ayumi Shiro, Takahisa Shobu, Yuji Higo, Mark H. Reid, Hiroshi Suzuki, Koichi Akita, \\ Ken-Ichi Funakoshi, and Klaus-Dieter Liss
}




\section{Lattice parameter evolution during heating of Ti-45Al-7.5Nb-}

\section{$2 \quad \mathbf{0 . 2 5} / \mathbf{0 . 5 C}$ alloys under atmospheric and high pressures}

$3 \mathrm{Xi} \mathrm{Li}^{\mathrm{a}, \mathrm{b}}$, Rian Dippenaar ${ }^{\mathrm{a}}$, Ayumi Shiro ${ }^{\mathrm{c}}$, Takahisa Shobu ${ }^{\mathrm{d}}$, Yuji Higo $^{\mathrm{e}}$, 4 Mark Reid $^{\mathrm{a}, \mathrm{b}}$, $\quad$ Hiroshi Suzuki $^{\mathrm{f}}, \quad$ Koichi Akita $^{\mathrm{f}}, \quad$ Ken-Ichi Funakoshi ${ }^{\mathrm{g}}$,

$5 \quad$ Klaus-Dieter Liss ${ }^{\text {a,b,f,h,i* }}$

$7{ }^{\mathrm{a}}$ School of Mechanical, Materials, Mechatronic and Biomedical Engineering, University of 8 Wollongong, NSW 2522, Australia

$9 \quad{ }^{\mathrm{b}}$ Australian Nuclear Science and Technology Organisation, Lucas Heights, NSW 2234, Australia

$10{ }^{\mathrm{c}}$ Quantum Beam Science Research Directorate, National Institute for Quantum and Radiological 11 Science and Technology, Kouto, Sayo, Hyogo 679-5148, Japan.

$12{ }^{\mathrm{d}}$ Materials Sciences Research Center, Japan Atomic Energy Agency, Kouto, Sayo, Hyogo 679-5148, 13 Japan.

$14{ }^{\mathrm{e}}$ SPring-8, Japan Synchrotron Radiation Research Institute, Kouto, Sayo, Hyogo 679-5198, Japan.

$15{ }^{\mathrm{f}}$ Materials Sciences Research Center, Japan Atomic Energy Agency, Tokai, Ibaraki 319-1195, Japan.

$16{ }^{\mathrm{g}}$ Neutron Science and Technology Center, Comprehensive Research Organization for Science and 17 Society (CROSS), Tokai, Ibaraki 319-1106, Japan.

$18 \quad{ }^{\mathrm{h}}$ Guangdong Technion - Israel Institute of Technology, Shantou 515063, China.

$19{ }^{\mathrm{i}}$ Technion - Israel Institute of Technology, Haifa 32000, Israel.

21 *Correspondence: kdl@gtiit.edu.cn, kdliss@technion.ac.il and liss@kdliss.de;

23 Abstract

24 Lattice strain evolution during deformation processing of Ti-Al alloys at high 25 temperature is important in terms of its microstructural evolution and microstructural 26 stability. It is shown here that careful evaluation of lattice parameters is critical for the 
27 understanding of thermal expansion, crystallographic order, chemical composition

28 and response to pressure, allowing to identify phase transitions and segregation, in

29 addition to the measurement of the more conventional quantities phase composition

30 and order parameter. The lattice parameters of Ti-45Al-7.5Nb-0.25/0.5C (at. \%) alloys

31 were calculated using both Rietveld and single peak fitting methods from data

32 obtained by in-situ synchrotron diffraction experiments at high temperature under

33 atmospheric and high pressure respectively. The lattice strain evolution as a function

34 of temperature in a Ti-45Al-7.5Nb-0.25C (at. \%) alloy under high pressure was

35 compared with that of a Ti-45Al-7.5Nb-0.5C (at. \%) alloy heated under atmospheric

36 pressure. The contribution of each of the four lattice strain factors is semi-

37 quantitatively assessed in the temperature range investigated.

39 Keywords: synchrotron radiation; TiAl; high pressure; high temperature; lattice strain

41 1. INTRODUCTION

42 Titanium aluminides are attractive candidate materials for applications in the

43 automotive industry, and more importantly for applications at high temperature in

44 aerospace industries, mainly due to their low density and excellent mechanical

45 properties [1-3]. A new route for the processing of titanium aluminide components

46 under high pressure has been proposed [4], for example, by utilizing a $0.8 \mathrm{GN}$ forging

47 press to manufacture large aerospace products [5] or a new $0.54 \mathrm{GN}$ die-forging press

48 currently being commissioned [6]. Since these forming processes operate at elevated

49 temperature and pressure, it is imperative that the microstructural integrity of the

50 work-pieces be maintained, especially by limiting grain growth during high- 
51 temperature processing and minimizing the development of internal stresses during 52 forging operations.

53 Processing in the $\left(\alpha_{2} / \alpha+\gamma\right)$ phase-field remains a challenge due to crystalline 54 anisotropy and the presence of intermetallic bonds. Appel et al. [7] pointed out that: 55 "The important difference with alloys that solidify completely through $\beta$ and those 56 that subsequently precipitate $\alpha$ on further cooling, is that the $\alpha$ that forms from the $\beta$ 57 can do so with up to 12 different orientation variants". Thus, a large $\beta$ grain can be 58 divided into lamellar colonies with up to 12 different orientations. This effect, termed 59 "crystal partitioning" [8,9], can therefore lead to significant grain refinement of 60 castings and significantly reduced texture. The $\beta$-phase is highly isotropic and 61 independent slip systems can operate during dynamic recovery as experimentally 62 confirmed by Liss et al. [10] in an in-situ synchrotron X-ray study. The $\beta$-solidifying 63 alloys being studied in the present program have a fine and homogenous 64 microstructure and are therefore easier to forge than conventional $\left(\alpha_{2} / \alpha+\gamma\right)$ alloys [7, $6511,12]$. It is possible to stabilize the $\beta$-phase by the addition of selected alloying 66 elements, but it has recently been established that the $\beta$-phase can also be physically 67 induced by the application of high hydrostatic pressure at high temperature. However, 68 the question as to how this newly formed $\beta$-phase would affect the lattice strains in 69 the $\alpha_{2} / \alpha$ and $\gamma$ phases has not been answered [13] and it is important to answer this 70 question because the lattice strains have a determining influence on the mechanical 71 properties of the product. Moreover, the evaluation of lattice parameters is a very 72 sensitive measurement to determine phase transformations of various kinds [14-16] 73 and can reveal segregation pathways in phase diagrams [17]. Therefore, under the 74 aspect of lattice parameter evolution as the main complementary evaluation we re- 
75 visit data from two in-situ heating experiments, to compare (i) a high-energy 76 synchrotron radiation study on Ti-45Al-7.5Nb-0.5C under atmospheric pressure [16]

77 with (ii) an energy-dispersive synchrotron X-ray diffraction experiment on Ti-45Al$78 \quad 7.5 \mathrm{Nb}-0.25 \mathrm{C}$ under high pressure at $9.6 \mathrm{GPa}[13]$

79 Figure 1(a) shows a section through the Ti-Al binary phase diagram [18], while a 80 section through a Ti-Al-7.5 at. \% Nb alloy, proposed by Chladil et al. [19] is shown in 81 Figure 1(b). The alloy used in the present study is schematically shown by the 82 vertical line at 45 at. $\% \mathrm{Al}$. It is important to note that this section through the phase 83 diagram applies to atmospheric pressure and to the knowledge of the authors, the 84 extent to which pressure changes the pertaining phase equilibria has not been 85 determined as yet.
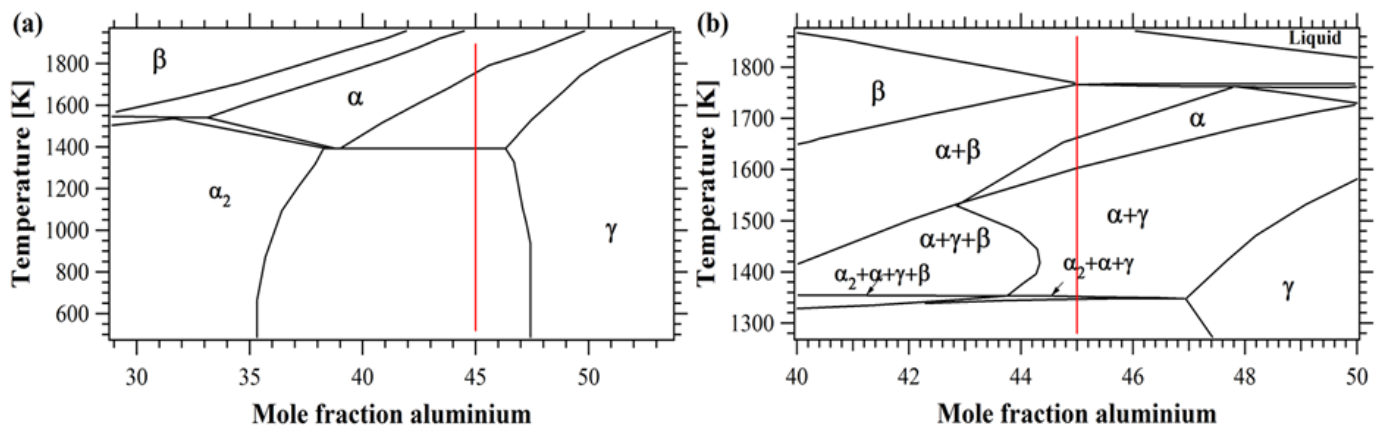

Figure 1. (a) Binary phase diagram of Ti-Al [18]; (b) Section through a proposed phase diagram of the Ti-Al-Nb alloy system for an alloy containing 7.5 at. \% $\mathrm{Nb}[19]$.

90 Since we study a Ti-Al-Nb-C alloy under conditions of severe-plastic deformation and 91 high temperature, it is important to identify the most critical parameters that 92 determine microstructural evolution and stability. One important variable is lattice 93 parameter evolution, since for example, a change in the $c / a$ ratio of the $\alpha$-lattice has a 94 determining influence on the pertaining slip and twinning deformation mechanisms. 95 Moreover, changes in the lattice parameter impact on orientation relationships and 
96 have an influence on interphase stress development. In addition, the mechanism and 97 morphology of phase transformations need to be taken into account since they play an 98 important role in achieving microstructural stability.

99 Although the lattice parameter evolution plays a pivotal role in assessing the high100 temperature behavior of titanium-aluminides, as argued above, only little information 101 has been traced to date, with the notable exception of the in-situ studies early by Shull 102 et al. [20], then by Yeoh et al. [21] and more recently that of Liss et al. [13].

103 The very early work of Shull et. al in 1990 reports on the first in-situ investigation of 104 titanium aluminides at high temperature, focusing on the experimental determination 105 of phase fields, while lattice parameter evolution is traced. In 2007, Yeoh et al. [21] 106 reported the changes occurring in the $c / a$ ratio of the lattice parameters in a Ti-45Al$107 \quad 7.5 \mathrm{Nb}-0.5 \mathrm{C}$ alloy during heating at atmospheric pressure. She suggested that as far as 108 X-ray analyses are concerned, a simplification should be made by assuming that the $109 \alpha_{2^{-}}$(ordered) and the $\alpha$-phase (disordered) be regarded as a single phase since X-rays 110 cannot clearly distinguish between an ordered and disordered structure.

111 Liss et al. [13] recently conducted an in-situ X-ray diffraction experiment on a Ti$11245 \mathrm{Al}-7.5 \mathrm{Nb}-0.25 \mathrm{C}$ alloy under high hydrostatic pressure. They studied the phase 113 evolution of a Ti-45Al-7.5Nb-0.25C alloy as a function of time under high pressure 114 and high temperature within a synchrotron X-ray source (SPring-8 beamline BL04B1, 115 run number M1472). The in-situ diffactograms are displayed in Figure 2 which have 116 been analyzed by the Rietveld method using MAUD (Material Analysis Using 117 Diffraction software $[22,23])$ for the evolution of phase fractions as a function of 118 temperature, shown in Figure 3. Also shown are the phase fractions determined by 119 Yeoh et al. [21] in a roughly similar alloy Ti-45Al-7.5Nb-0.5C, but under standard 
120 atmospheric conditions. The two alloys have been manufactured under identical

121 conditions and the two in-situ experiments were conducted under normal atmospheric

122 and high pressure respectively. However, it is important to note that carbon can have a

123 significant influence on phase evolution in these alloys and for this reason care need

124 to be taken in comparing the alloys containing 0.25 at. $\% \mathrm{C}$ and 0.5 at. $\% \mathrm{C}$

125 respectively. For example, the eutectoid temperature, $T_{\text {eu }}$, is increased by $20 \mathrm{~K}$ from

$1261453 \mathrm{~K}$ to $1473 \mathrm{~K}$, but the respective $\gamma$-solvus, $T_{\gamma \text {,solv }}, 1565 \mathrm{~K}$ and $1566 \mathrm{~K}$ respectively

127 [24] remains essentially constant. Notwithstanding these differences, the two alloys

128 can be compared with respect to their respective pressure-induced behaviors. The $f_{c c}$ -

129 based, ordered $\gamma$-phase of $\mathrm{L}_{0}$ structure, co-exists with an $h c p$-based, ordered $\alpha_{2}$-phase

130 of $\mathrm{D}_{19}$ structure at room temperature. Upon heating, the $\alpha_{2}$-phase undergoes an

131 inverse eutectoid order-disorder transition to form a fully disordered hexagonal $\alpha$ -

132 phase at $T_{\text {eu }}$. The fraction of the $\gamma$-phase decreases upon heating and finally transforms

133 fully into the disordered $\alpha$-phase at $T_{\gamma, \text { solv }}$. Salient features when the Ti-45Al-7.5Nb-

$1340.25 \mathrm{C}$ alloy is heated under a pressure of $9.6 \mathrm{GPa}$, are the appearance of the $b c c \beta$ -

135 phase in an $\left(\alpha+\alpha_{2}+\beta+\gamma\right)$ field and the dissolution of $\gamma$ phase at $T_{\gamma, \text { solv }}$ to form $136 \quad(\alpha+\beta)$. 


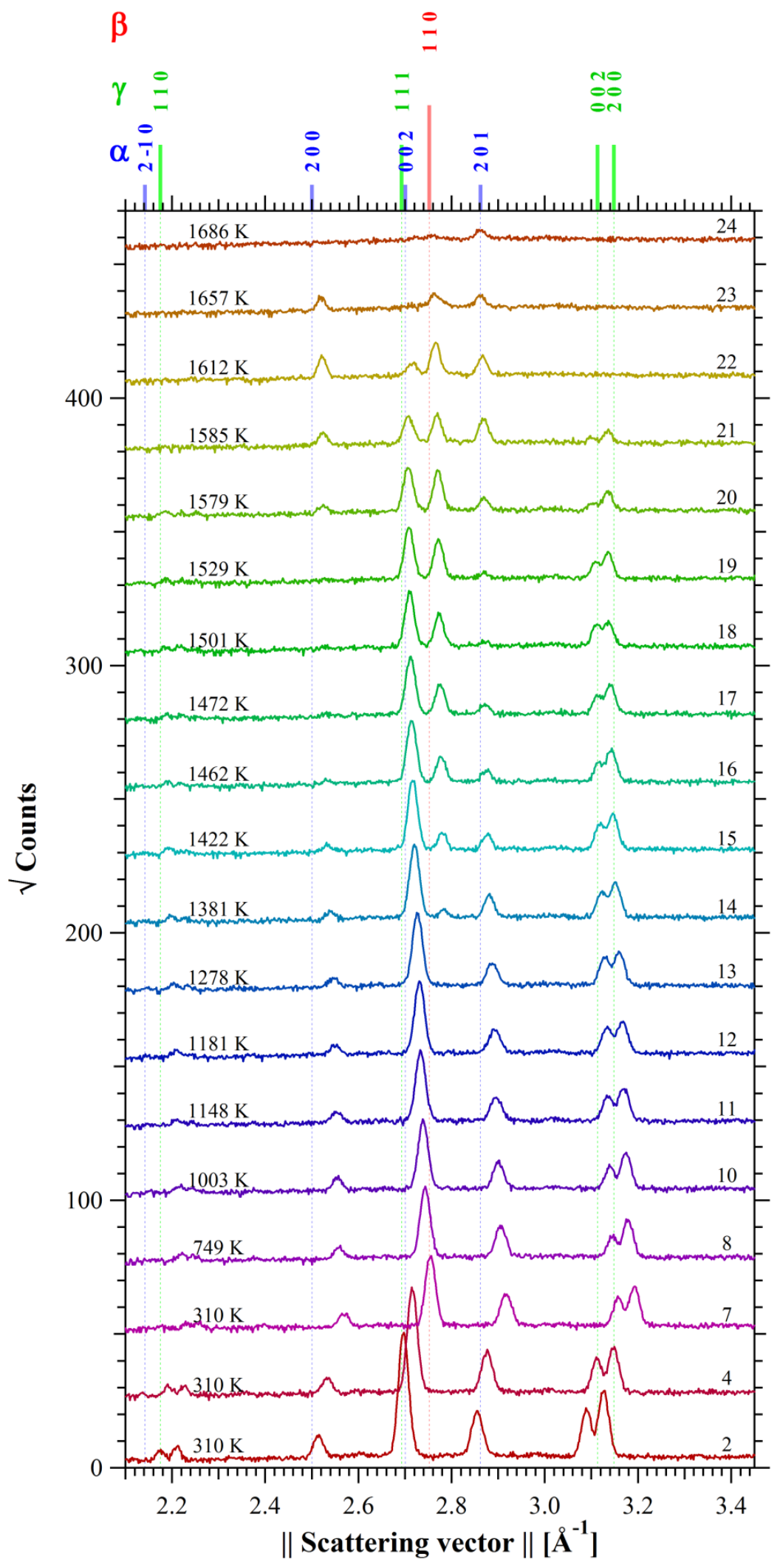

138 Figure 2. Measured diffraction patterns of a Ti-45Al-7.5Nb-0.25C alloy obtained under high pressure (9.6 GPa). Temperature tags are shown on the left and serial numbers on the right [13]. The first three patterns at $310 \mathrm{~K}$ were taken at pressures of $0,3.2$ and $9.6 \mathrm{GPa}$, respectively (based on [13] under CCBY license). 


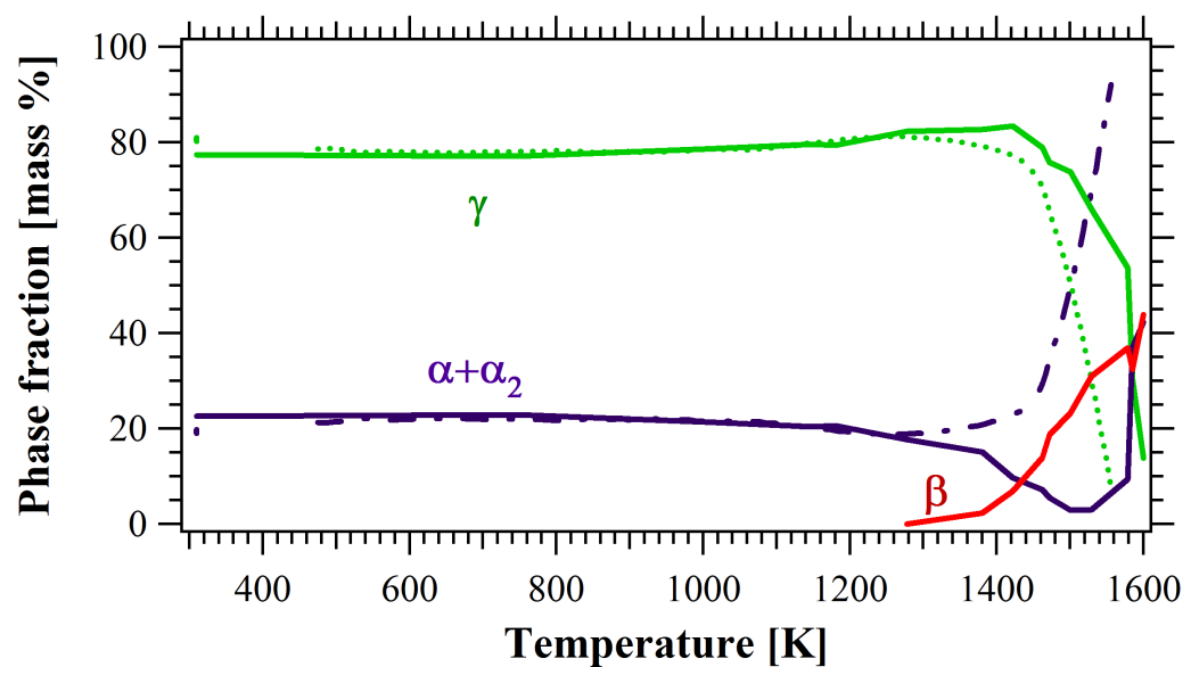

145 Figure 3. Phase evolution in a Ti-45Al-7.5Nb-0.25C alloy at a pressure of 9.6 GPa (continuous lines) 146 (based on [13] under CC-BY license) compared with observations at standard atmospheric pressure 147 (dotted lines) for a Ti-45Al-7.5Nb-0.5C alloy, replotted from Yeoh et al. [21].

149 Liss et al. [13] analyzed the lattice strain development in the Ti-45Al-7.5Nb-0.25C 150 alloy at $310 \mathrm{~K}$ at three pressures, up to $9.6 \mathrm{GPa}$ (series numbers 2, 4 and 7 in Figure 1512 2). They calculated the changes in lattice parameter of the $\gamma$ - and $\alpha_{2} / \alpha$-phases as a 152 function of pressure at this temperature [13] and argued that at room temperature, the 153 volume response to pressure is accommodated by the phase transformation $\gamma \rightarrow \alpha_{2}$, 154 rather than by volumetric strain. They further determined some crystallographic 155 aspects, specifically lattice strain and atomic order, at room temperature, but did not 156 determine lattice strain evolution during heating at high pressure, which is subject of 157 the current project. It is the dearth of information of this parameter, critical to 158 processing at high temperature and pressure, which prompted the present 159 investigation.

160 The overarching aim of the present work was the re-visit of the experimental data of 
161 both experiments [13, 27] in order to compare the behavior of the selected alloys

162 under atmospheric and high pressure respectively.

163 The specific aims were:

164 - to determine the lattice parameter evolution as a function of temperature at 165 atmospheric pressure in the Ti-45Al-7.5Nb-0.5C alloy.

166 - to determine lattice parameter evolution as a function of temperature under high 167 hydrostatic pressure in the Ti-45Al-7.5Nb-0.25C alloy.

168 It will be shown below that the experimentally determined lattice parameter 169 evolutions occur in response to thermal expansion, alloy element segregation, order 170 parameter and pressure. The specific trends and values will be decomposed based on 171 an understanding of strain evolution with reference to the reported phase diagram at 172 atmospheric pressure, before interpreting the evolution in the unknown system under 173 high pressure. These new insights are opening new pathways to better understand 174 structural transformations in the experimentally confined system. Such understanding 175 is an essential element in optimizing the intended production techniques of titanium176 aluminides since microstructural stability plays an important role in determining 177 processing parameters.

178

179 2. EXPERIMENTS

180 Lattice strain evolutions were calculated from the raw data of two earlier experiments:

181 - Yeoh et al. [21] conducted in-situ high-energy X-ray diffraction studies under 182 proposal number MA-77 at the ID15B beamline at the ESRF in Grenoble using a 183 two-dimensional detector. They ramped up a Ti-45Al-7.5Nb-0.5C alloy from 
room temperature to $1375 \mathrm{~K}$ at a rate of $5 \mathrm{~K} / \mathrm{min}$ before ramping down to a rate of $2 \mathrm{~K} / \mathrm{min}$. Once a temperature of $1675 \mathrm{~K}$ was reached, it was maintained for 5 minutes before cooling down to room temperature at a rate of $5 \mathrm{~K} / \mathrm{min}$. All details of the experiment have been described by Yeoh et al. [21].

- Liss et al. [13] conducted in-situ X-ray diffraction studies at the BL04B1 beamline at the modern synchrotron source SPring-8 $[25,26]$ and a detailed

Both single peak fitting and Rietveld refinement, using the MAUD program, were

204 conducted to extract lattice parameters and phase fractions from the diffraction 205 patterns obtained in these two experiments. The Igor Pro 4 software (WaveMetrix, 206 Lake Oswego, OR, USA) was then used to calculate the lattice strains and to perform 207 curve fitting. 
208 The two titanium-aluminides of nominal chemical composition Ti-45Al-7.5Nb-

$2090.25 / 0.5 \mathrm{C}$ used in the present study were produced by a powder metallurgical

210 processing technique. Powder was manufactured by a plasma-melting induction-

211 guiding atomization technique and consolidated by hot-isostatic pressing for $2 \mathrm{~h}$ at a

212 pressure of $200 \mathrm{MPa}$ and a temperature of $1553 \mathrm{~K}$ [27]. Both alloys were

213 characterized by Chladil et al. [4, 24] and the resulting microstructure at room

214 temperature consists of a globular $\gamma$-phase and lamellar $\left(\alpha_{2}+\gamma\right)$ two-phase colonies

$215 \quad[4]$

216 While the experimental settings are described in the references, we like to raise an

217 error estimate at this point, particularly to validate the later described lattice parameter

218 fluctuations and features of the present manuscript. While the atmospheric pressure

219 data in angle-dispersive setting appear as smooth curves, due to much faster and

220 therefore finer sampling, the high-pressure data has been undertaken on temperature

221 holding steps with larger step size, representing sometimes larger jumps between

222 them, which could be interpreted as error fluctuations. Care has been undertaken by

223 validating the realty of such jumps re-visiting the original data - i.e. looking out for

224 changes of peak shape, overlapping etc and by the two kinds of fitting analysis both

225 Rietveld and single peak. We come up with the following estimate of error,

226 demonstrating the trend of the features we discuss on lattice strain evolution:

- For the high-pressure setup [13] the energy calibration based on fluorescence lines of $\mathrm{Mo}, \mathrm{Pb}, \mathrm{Au}, \mathrm{Ag}, \mathrm{Pt}, \mathrm{Ta}$ and $\mathrm{Cu}$ and single peak fitting (see below) has been calibrated to and accuracy of $\sim 1 \mathrm{E}-4$. Subsequently, the diffraction angle was calibrated using $\mathrm{MgO}$ and $\mathrm{Au}$ as standard materials at ambient conditions.

- At $1278 \mathrm{~K}$, the single Gaussian fitted absolute peak positions for the $[\gamma$ $\left.111, \alpha_{2}-201\right]$ reflections are $[2.73,2.89] \AA^{-1}$ (we reproduce only 3 digits in this 
text, however error propagation used full numerals) with an error of [5.49E-5, $1.8 \mathrm{E}-4] \AA^{-1}$ resulting in $\Delta G / G$ of $[2.0 \mathrm{E}-5,6.2 \mathrm{E}-5]$. Similar results are obtained at $1612 \mathrm{~K}$, also for the $\beta-110$ reflection with a value of $\Delta G / G=3.9 \mathrm{E}-5$.

- Moreover, we are interested in strain values rather than absolute values which propagates the errors and evaluates to double the $\Delta G / G$ uncertainties, resulting in about $\sim(1-2)$ E-4.

- Rietveld refinement reported inaccuracies of lattice parameter determinations as represented in the subsequent plots. They are mostly consistent with the error estimation by single peak fitting, however Rietveld error bars diverge for the $\alpha_{2} / \alpha$-phase around $1500 \mathrm{~K}$ because of the weakness of some of their peaks.

244 or pressure, as it will be discussed further in the paper. We exclude significant drifts 245 during a particular holding step, which would otherwise express in peak shape and 246 broadening. Single-peak fitting of the $\alpha_{2}$-201 reflection has been performed and con247 firms the trends with an error of $\sim 3 \mathrm{E}-4$, even in the range around $1500 \mathrm{~K}$, allowing a 248 qualitative interpretation of peak shifts due to various lattice variations, such as 249 change of phase composition, disorder transformation etc, as it will be discussed.

\section{RESULTS}

\subsection{Lattice strains at standard atmospheric pressure}

253 The evolving lattice strains were calculated by equation (1),

$$
\varepsilon=\varepsilon_{h k l}=\left(G_{0}-G\right) / G_{0}
$$

255 For a given reflection, $G_{0}$ are the reciprocal lattice vectors at $300 \mathrm{~K}$ and $G$ are the 256 measured values at increasing temperature. The lattice strain discussed below is not 257 an absolute lattice strain but is merely the difference in strain with respect to a 258 reference value at $300 \mathrm{~K}$. Lattice strains of the Ti-45Al-7.5Nb-0.5C alloy at standard 
259 atmospheric pressure are shown in Figures 4 and 5 for the co-existing phases, $\alpha_{2} / \alpha$

260 and $\gamma$, respectively. Both figures display an initial linear relationship between lattice

261 strain and temperature which then evolves abnormally and anisotropically. Also

262 shown is the average lattice strain, expressed as $\Delta V / 3 V$ of the atomic volume

263 expansion $(\Delta V / V=2 \Delta a / a+\Delta c / c)$, as a function of temperature. The changes in

264 lattice strain can be divided into four distinct temperature regions, which we discuss

265 for each phase.

\subsubsection{The $\alpha_{2} / \alpha$-phase}

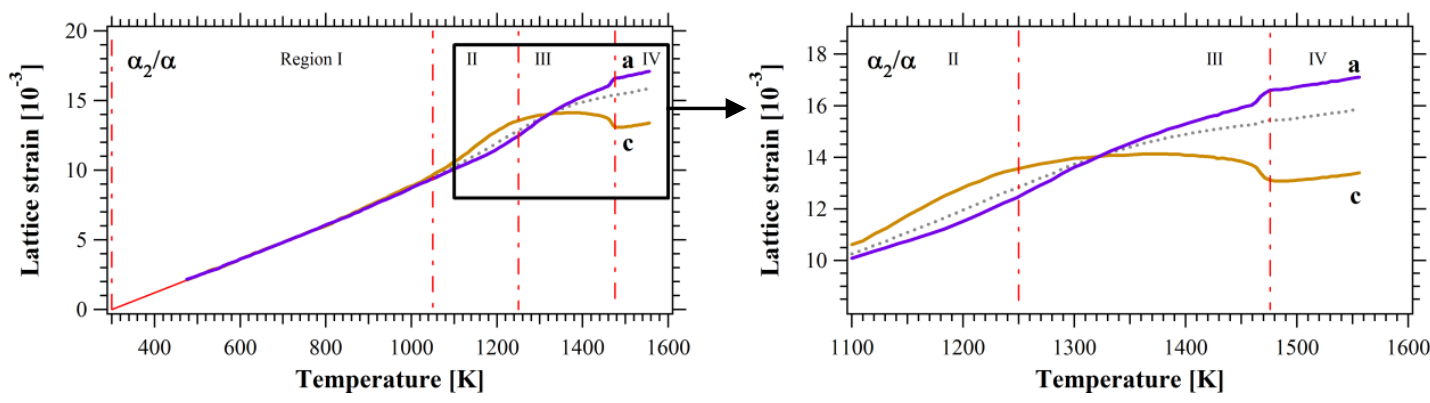

Figure 4. Dependence of the lattice strains in the $\alpha_{2} / \alpha$-phase along the $a$ - and $c$-directions (purple and brown respectively) as well as $1 / 3$ of volumetric expansion (dotted curve), as a function of temperature

272 In region I, strain evolution in both $a$ - and $c$-directions is linear. In region II, the

273 strains increase gradually to higher values than can be accounted for by linear thermal

274 expansion alone. The lattice strain along the $a$-direction in region III increases more

275 rapidly than in region II whereas it remains nearly constant along the $c$-direction, even

276 under the influence of the counter-balanced thermal expansion. Region IV is

277 characterized by a distinct kink in the strains of both lattice parameters at $1476 \mathrm{~K}$

278 [21], presumably as a result of the occurrence of the reverse eutectoid reaction $\alpha_{2} \rightarrow$ 
$279 \alpha$. Following these sudden changes, the strain increases gradually with an increase in 280 temperature.

\subsubsection{The $\gamma$-phase}

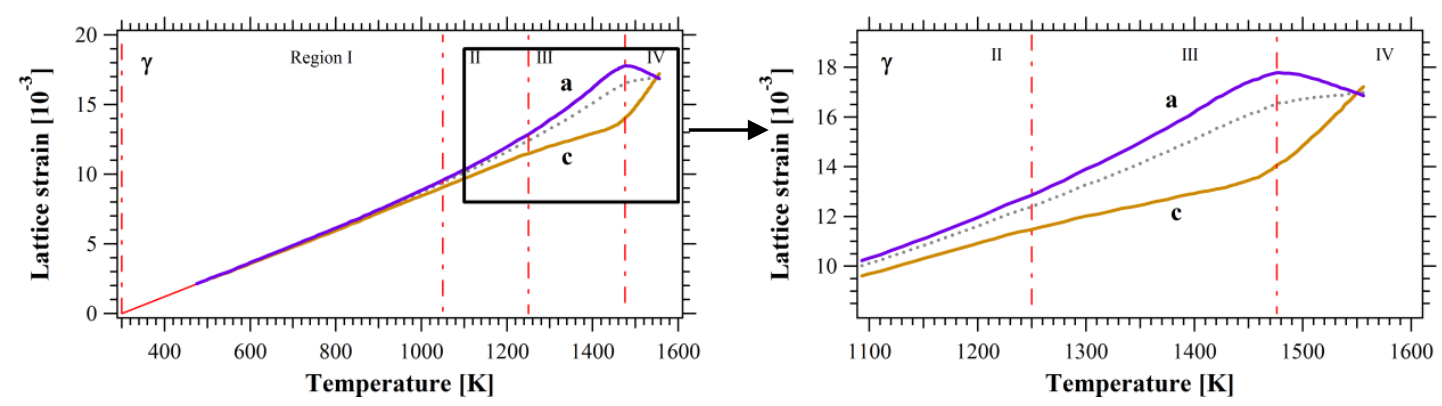

Figure 5. Lattice strains in the $\gamma$-phase along the $a$ - and $c$-directions (purple and brown) as well as $1 / 3$ of the volumetric expansion (dotted curve), as a function of temperature in a Ti-45Al-7.5Nb-0.5C alloy at standard atmospheric pressure.

The lattice strain evolution of the $\gamma$-phase is shown in Figure 5 in both the $a$ - and $c$ -

288 directions, together with the weighted average (1/3 of the volumetric strain).

289 In region $\mathrm{I}$, the strain evolution of the $\gamma$-phase is similar to that in the $\alpha_{2} / \alpha$-phase. In

290 region II, the strain in both the $a$ - and $c$-directions increases almost linearly since the

291 dominant contributing factor to lattice strain is thermal expansion. In region III, the

292 strain in the $\gamma$-phase along the $a$-direction increases up to $T_{\text {eu }}$ whereas the strain in the $293 c$-direction increases to a lesser extent, developing significant anisotropy. In region IV,

294 the strain in the $a$-direction decreases while the strain in the $c$-direction increases. The 295 volumetric strain deviates only slightly from linearity and at the $T_{\gamma, \text { solv }}$ of $1565 \mathrm{~K}$, all 296 strain components meet as if they had expanded isotropically and linearly from room 297 temperature. 
300 The experimentally determined lattice strains of the $\alpha_{2} / \alpha$ - and $\gamma$-phases are shown in

301 Figures 6 and 7 for the Ti-45Al-7.5Nb-0.25C alloy under a pressure of 9.6 GPa. It is

302 instructive to divide strain development into four regions.

303 Region I depicts the lattice strain evolution as a function of temperature in the range

$304300 \mathrm{~K}$ to $1003 \mathrm{~K}$, where the $\alpha_{2}$-phase has not yet reached thermodynamic equilibrium.

305 Region II ranges from $1003 \mathrm{~K}$ to $1420 \mathrm{~K}\left(T_{\gamma, \max }\right)$ and is divided into sub-regions $\mathrm{II}_{\mathrm{a}}$

306 and $\mathrm{II}_{\mathrm{b}}$, separated by the appearance of the $\beta$-phase at $1350 \mathrm{~K}\left(T_{\beta, \text { start }}\right)$ [13]. Region III

307 ranges from $1420 \mathrm{~K}$ to $1510 \mathrm{~K}\left(T_{\text {eu }}\right)$, while region IV covers the temperature range

308 above $1510 \mathrm{~K}$.

309

\subsubsection{The $\alpha_{2} / \alpha$-phase}

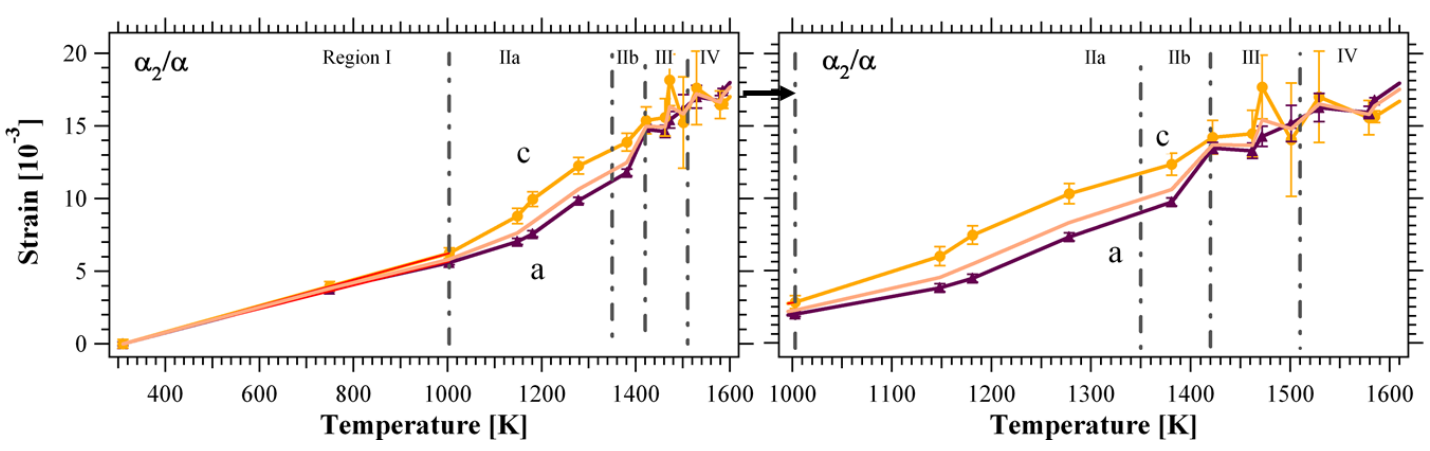

Figure 6. Lattice strains in the $\alpha_{2} / \alpha$-phase along $a$-(purple), $c$-(brown) directions and $1 / 3$ of volumetric expansion (pink) as a function of temperature $(\mathrm{K})$ for a Ti-45Al-7.5Nb-0.25C alloy at a nominal pressure of $9.6 \mathrm{GPa}$.

316 Figure 6 shows the lattice strain evolution of the $\alpha_{2} / \alpha$-phase along the $c$ - and $a$ -

317 directions respectively. In region I, the lattice strain evolution is linear with respect to

318 temperature. The strains along the $a$ - and $c$-directions in region II increase more than 
319 those in region $\mathrm{I}$. In region $\mathrm{II}_{\mathrm{b}}$, the slope of the strain-temperature curve along the $a$ -

320 direction is higher than that along the $c$-direction, indicating a decrease in the $c / a$ -

321 ratio. A significant increase in strain occurs at $1420 \mathrm{~K}$ (the $T_{\gamma, \max }$ ). In region III, a

322 maximum value of the lattice strain along $c$-direction is noticed at $1472 \mathrm{~K}$. In region

$323 \mathrm{IV}$, the lattice strain increases in the $a$-direction while there is an erratic behavior in

324 the $c$-direction.

\subsubsection{The $\gamma$-phase}

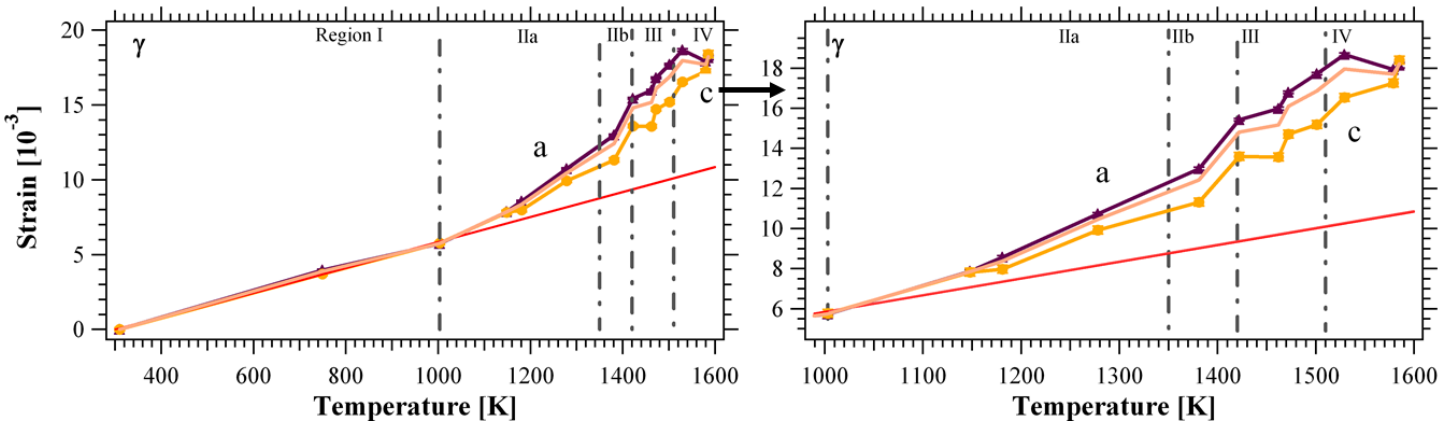

Figure 7. Lattice strain in the $\gamma$-phase along $a$ - (purple), $c$ - (brown) directions and $1 / 3$ of volumetric expansion (pink curve) as a function of the temperature $(\mathrm{K})$ for Ti-45Al-7.5Nb-0.25C at a nominal pressure of $9.6 \mathrm{GPa}$.

331 Figure 7 illustrates the lattice strain evolution of the $\gamma$-phase in the $a$-and $c$-directions,

332 respectively. Noticeably, in region $\mathrm{II}_{\mathrm{a}}$, the strain increases more along the $a$-direction

333 than in the $c$-direction, leading to a decrease of the $c / a$-ratio. In region $\mathrm{II}_{\mathrm{b}}$, the strain

334 continues to increase but more so than in the previous region. In region III, the strain

335 increases but not as steeply as in the region $\mathrm{II}_{\mathrm{b}}$. In region $\mathrm{IV}$, the strain evolution trend

336 is almost the same as under standard atmospheric pressure. The transformation

337 temperatures $T_{\text {eu }}$ and $T_{\gamma, \text { solv }}$ shift to higher temperatures at high pressure, evidenced by

338 a comparison between Figures 5 and 7. 


\subsubsection{Experimental pressure release}

340 During the experiments conducted at a hydrostatic pressure of $9.6 \mathrm{GPa}$, we have

341 observed a progressive loss of sample pressure as a result of softening of the gaskets

342 of the pressure cell at increasing temperature. Therefore, the sample pressure creeps

343 down to about $6.9 \pm 0.5 \mathrm{GPa}$ over the time of the experiment, measured at $1473 \mathrm{~K}$.

344 This pressure loss of about $2.7 \mathrm{GPa}$ has a marked effect on the absolute value of the

345 measured crystal strain. In order to estimate the extent to which this pressure creep

346 affects the quantitative values of the measured strain, we conducted the following

347 analysis:

348 Experiments conducted at standard atmospheric pressure show that the volumetric

349 lattice expansion increases linearly with temperature as shown in Figures 4 and 5.

350 However, when the experiments are conducted at high pressure, there is a significant

351 deviation from linearity to larger strain values, as shown in Figures 6 and 7. This

352 deviation is illustrated in Figure 7 where the red line shows the extrapolation of linear

353 thermal expansion.

354 There is clearly a significant difference between this expected linear dependence of

355 lattice strain development as a function of temperature and the experimentally 356 observed volumetric values (pink curve). We believe that this deviation is not due to 357 differences in lattice strain, but is caused by thermally induced softening of the 358 gaskets of the pressure cell as outlined above. Under atmospheric pressure, the linear 359 extrapolation of thermal expansion meets closely the point of volumetric strain at $360 T_{\gamma, \text { solv }}$, as shown in Figures 4 and 5. In order to assess this premise, we measured the 361 difference in strain between the extrapolated linear thermal expansion and the average 362 strain expressed by $1 / 3$ volumetric strain (red and pink lines in Figure 7) at a 
363 temperature of $1579 \mathrm{~K}$ as 0.00702 . Since the value of the bulk modulus is known, 152

$364 \mathrm{GPa}$ [13], we used the relation $\partial p=-K \cdot \partial V / V$ to calculate the pressure difference

365 as $3.20 \mathrm{GPa}$. This calculated value of pressure release is in reasonable agreement with

366 the experimentally measured decrease of $2.7 \mathrm{GPa}$ in pressure measured following the

367 heating cycle, thereby providing evidence that the observed deviation can indeed be

368 attributed to the sample pressure release as a result of a leak in the seal of the pressure

369 cell. The two pressure release values are particularly in good agreement taking into

370 account that only the room temperature bulk modulus is available.

371 The values of strain shown in Figure 7 are therefore higher than the true values of

372 lattice strain under $9.6 \mathrm{GPa}$ by up to $25 \%$ and the isotropic lattice strain as a result of

373 temperature change has to be estimated. However, the anisotropic part, as will be 374 discussed later, is well representative.

\section{4. DISCUSSION}

376 In order to better understand how changes in lattice parameter influence 377 microstructural evolution, account has to be taken of the factors contributing to such

378 lattice strains. When the temperature increases, the strains in both crystallographic $a$

379 and $c$ directions increase, while an increase in the aluminium content in the respective

380 phase results in a decrease in those strains. The order parameter is defined as $\mathrm{S}=$

$381 \frac{p-r}{1-r}$, where $p$ is the probability that an atom resides in the ordered position $r$ (The 382 concept of the order parameter is discussed in more detail in the Appendix). The order 383 parameter is a measure of the anisotropic behavior: when either the $\alpha_{2}$ - or $\gamma$-phase 384 develops an ordered structure, the strain along the $a$-direction decreases whereas that 385 along the $c$-direction increases. A summary of the influence of the order parameter in 386 the different temperature regions, is shown in Figures 8, 9, 11 and 13. It is to be noted 
387 that these figures only show the trend in strain (the exact quantitative strain values 388 haven't been shown).

\subsection{Lattice strains at standard atmospheric pressure}

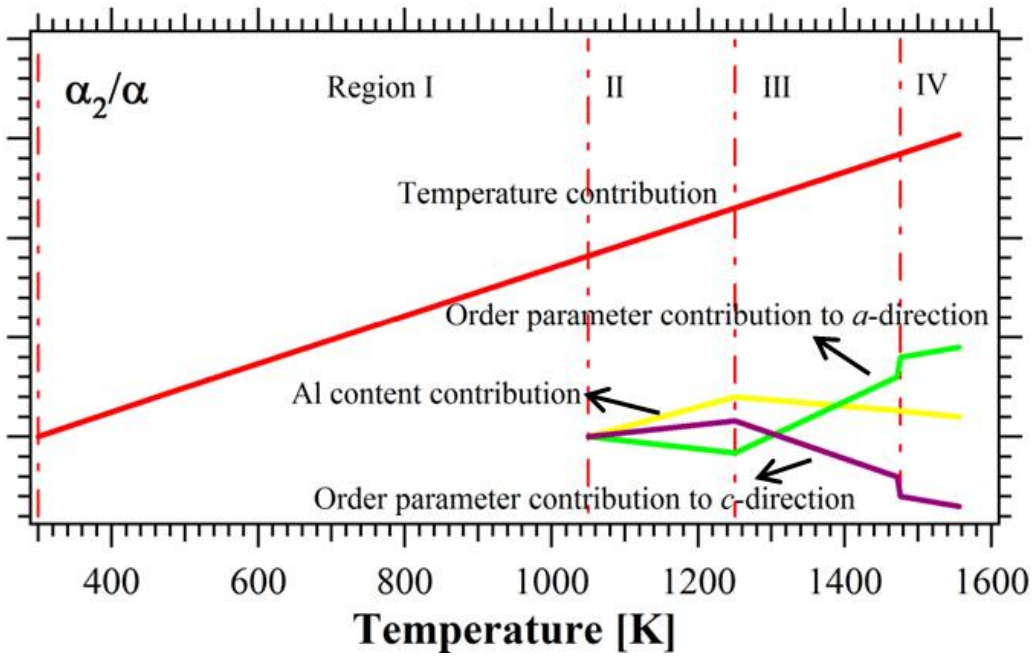

Figure 8. Factors contributing to lattice strain evolution in the $\alpha_{2} / \alpha$-phase at standard atmospheric pressure.

396 Figure 8 shows how temperature, aluminium content and the order parameter affect

397 strain evolution of the $\alpha_{2} / \alpha$-phase under standard atmospheric pressure. It is pertinent

398 to discuss these observations:

399 Region I: lattice strain increases linearly with respect to temperature because the 400 dominating contributing factor is thermal expansion, which, for most solids is 401 proportional to the temperature change $\Delta T$, with $\varepsilon_{\text {thermal }}=\eta \cdot \Delta T$ [28], where $\eta$ is 402 the linear thermal expansion coefficient. Region II: It is reasonable to assume that the 403 high-temperature equilibrium concentration of aluminium has been 'frozen' into the 404 alloy during manufacturing. This means that the aluminium concentration in $\alpha_{2}$ is 
405 effectively higher than the equilibrium concentration at room temperature. With an

406 increase in temperature, long-range diffusion of aluminium becomes possible, such

407 that the $\alpha_{2}$-phase is depleted in aluminium, effectively meaning that the titanium

408 concentration in the $\alpha_{2}$-phase increases. Higher concentrations of titanium lead to an

409 increase in atomic volume [13] and hence, accounts for the observed increase in

410 lattice strain as a function of temperature. Region III: The curvature of lattice strain

411 evolution in the $\alpha_{2} / \alpha$-phase along the $a$ - and the $c$-direction has different signs. This

412 observation is ascribed to the occurrence of an order-disorder transformation (the

413 order-disorder transformation is discussed in more detail in the appendix). Region IV:

414 Both lattice strains $\varepsilon_{a}$ and $\varepsilon_{c}$ show a distinct, respectively positive and negative step

415 change at the boundary of regions (III) and (IV), coinciding with a sharp decrease in

416 order parameter (see Figure A.2, Appendix). It is important to note that the volume

417 strain evolves smoothly, without a step-change. This observation provides evidence of

418 the premise that the change of order does not affect the atomic volume, but only strain

419 anisotropy.

420

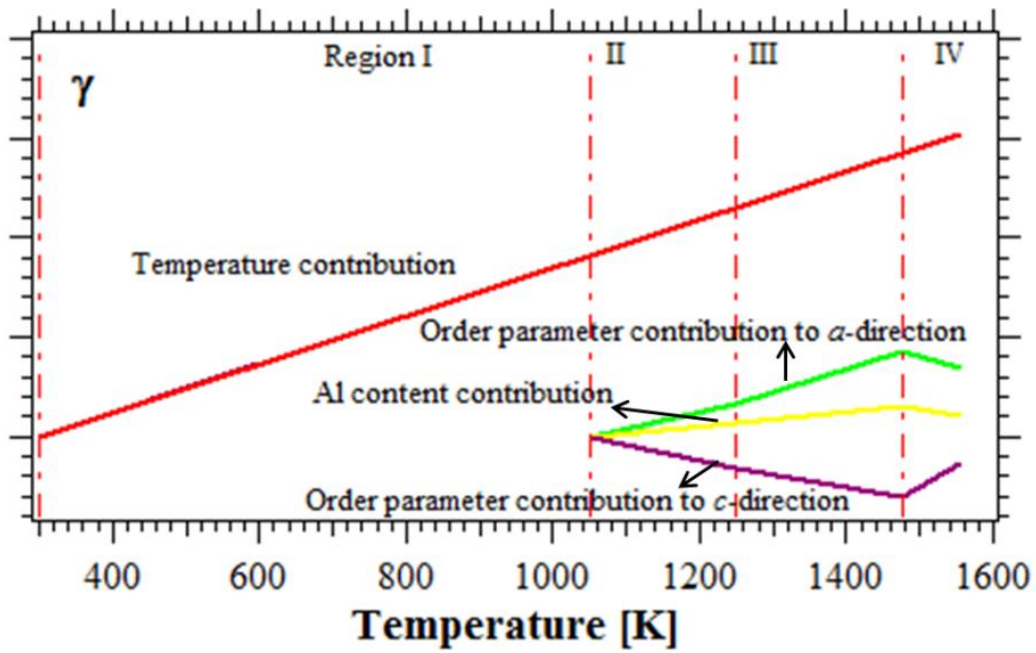

Figure 9. Factors contributing to lattice strain evolution in the $\gamma$-phase at atmospheric pressure. 
424 Figure 9 shows how temperature, aluminium content and the order parameter affect

425 strain evolution in the $\gamma$-phase at standard atmospheric pressure.

426 The thermal expansion is linear in region I along the $a$ - and $c$-directions due to the

427 linear temperature contribution. The small and anisotropic deviation from linearity in

428 Region II is due to the extent to which aluminium contributes to the disordering of the

429 TiAl-structure. It is observed that the lattice strain along the $a$-direction increases

430 faster than along the $c$-direction, and since the $c$-dimension is larger than the $a$ -

431 dimension, the unit cell approaches more closely an $f c c$ unit cell, that of a fully

432 disordered $\gamma$-phase, revealing a higher degree of disorder and hence, a smaller order

433 parameter. Witusiewicz et al. [29] have shown earlier that the aluminium

434 concentration decreases well below stoichiometry in this temperature range as a

435 function of temperature and that the lowest aluminium concentration in the $\gamma$-phase is

436 attained at $1476 \mathrm{~K}$, contributing to chemical disorder. Above $1476 \mathrm{~K}$ (in region IV),

437 the aluminium content in the partially ordered $\gamma$-phase increases, as shown in Figure

438 10(a). Yeoh et al. [21] have shown that the $c / a$ ratio decreases sharply with increasing

439 temperature in Region III as shown in Figure 10(b). Hence, the strain along the $a$ -

440 direction increases more than in the $c$-direction and the TiAl-structure becomes highly

441 disordered. In region IV, the $c / a$ ratio increases sharply as shown in Figure 10(b) and

442 the fully ordered TiAl-structure is approximated, due to the highest ordering energy of

443 the $\gamma$-phase, compared to all other Ti-Al configurations [30]. 

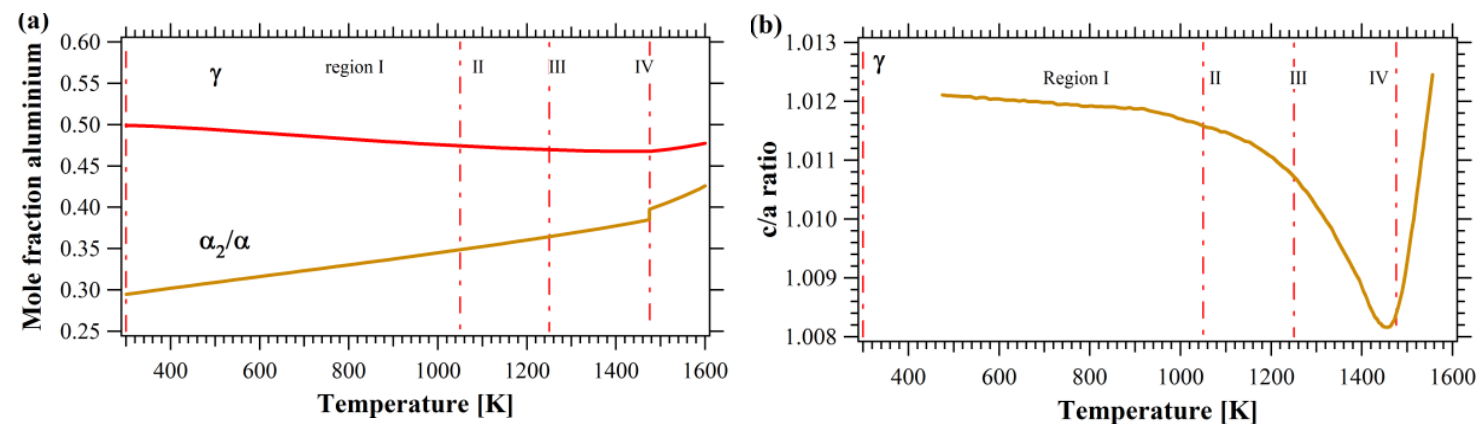

Figure 10: (a) Aluminium concentrations of the $\gamma$ - and $\alpha_{2} / \alpha$-phases as a function of temperature as dereived from the phase diagram [29], (b) The $c / a$ ratio of the $\alpha_{2} / \alpha$-phase for a Ti- $45 \mathrm{Al}-7.5 \mathrm{Nb}-0.5 \mathrm{C}$ alloy at standard atmospheric pressure [21].

\subsection{Lattice strains at high pressure}

449 Figure 11 shows the trend in the strain evolution by the respective contributions of

450 temperature, aluminium content in the respective phases, order parameter and

451 pressure in the different regions for the $\alpha_{2} / \alpha$-phase at a nominal pressure of $9.6 \mathrm{GPa}$.

452 In order to avoid bias from the experimental pressure release, we calculate the 453 anisotropic part of strain $(\Delta a / a-\Delta V / 3 V, \Delta c / c-\Delta V / 3 V)$ as shown in Figure 12 for

454 the $\alpha_{2} / \alpha$-phase. The main features of the strain evolution of the $\alpha_{2} / \alpha$-phase at $9.6 \mathrm{GPa}$ 455 are illustrated as follows:

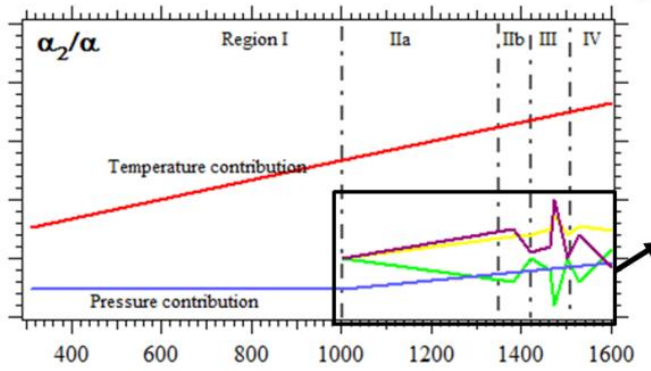

Temperature $[\mathrm{K}]$

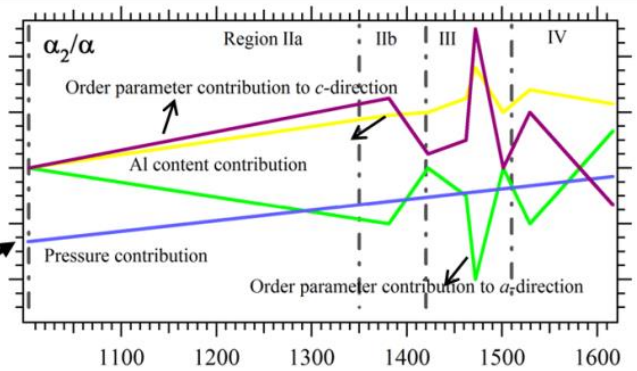

Temperature $[\mathbf{K}]$ 


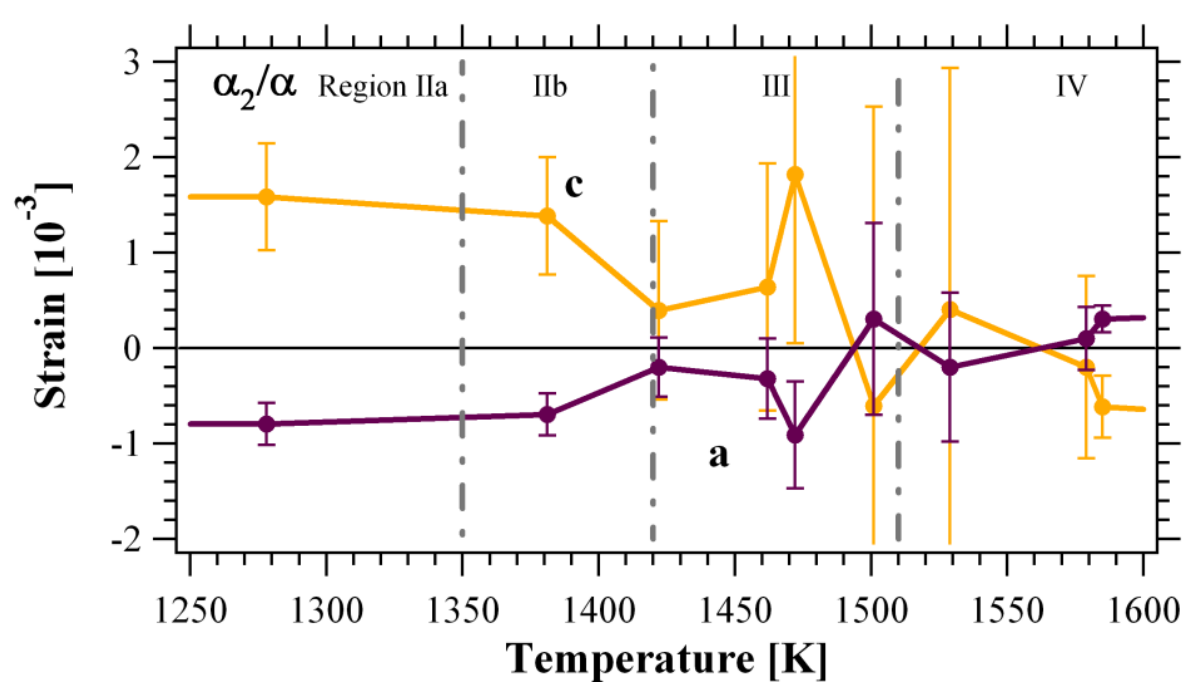

Figure 12. Anisotropic lattice strains of the $\alpha_{2} / \alpha$-phase along the $a$ - (purple) and $c$ - (brown) directions for the Ti-45Al-7.5 Nb-0.25C alloy at $9.6 \mathrm{GPa}$

466 In region $\mathrm{II}_{\mathrm{b}}$ the anisotropic strain increases in the $a$-direction and decreases in the $c$ -

467 direction. These observations are attributed to the order-disorder transition in the $\alpha_{2} / \alpha-$ 468 phase. In region III, the $\beta$-phase exists. The strain evolution under a pressure of $4699.6 \mathrm{GPa}$ is in the opposite direction in the temperature range $1420 \mathrm{~K}$ to $1472 \mathrm{~K}$ than 470 observed under standard atmospheric conditions at the beginning of region III (see 471 Figure 4). There is a steep increase along the $c$-direction between $1462 \mathrm{~K}$ and $1472 \mathrm{~K}$, 472 corresponding to the increase of the $\beta$-phase fraction (see Figure 3 ). The $\beta$-phase in 473 solid solution has a $b c c$ structure and provides an opportunity for the co-existing $\alpha_{2}-$ 474 phase to drive closer to stoichiometry and order, increasing its $c / a$ ratio. Moreover, 475 under the assumption that $\beta$ orders to $\beta_{0}$ with a B2 structure, the latter would extract 476 Al from $\alpha_{2}$, again emphasizing a higher degree of order in the latter. The 477 transformation to $\beta$ leads to a sharp decrease in the aluminium content of the 478 supersaturated $\alpha_{2} / \alpha$-phase and hence, the $\alpha_{2} / \alpha$-phase is increasingly ordered. The 479 change in the fraction of $\beta$-phase has a major influence on the aluminium 
480 concentration in the $\alpha_{2} / \alpha$-phase from region III onwards. These arguments explain 481 why the trend of the anisotropic strain in the $\alpha_{2} / \alpha$-phase correlate well with the $\beta$ 482 phase evolution. By contrast, the main contributor is a thermally driven $\alpha \rightarrow \alpha_{2}$ order483 disorder transition in region III under standard atmospheric pressure. The maximum 484 value of anisotropic strain along the $c$-direction at $1472 \mathrm{~K}$ is probably driven by the 485 appearance of an ordered $\beta_{0}$-phase, leading to a lower aluminium content in the $\alpha_{2} / \alpha$ 486 phase. Under a pressure of $9.6 \mathrm{GPa}, 1510 \mathrm{~K}$, as a minimum of $\alpha$-phase fraction, is 487 taken as eutectoid temperature, reported by Liss et al. [13]. It indicates that under high 488 pressure the eutectoid temperature would be increased. On approach to $1510 \mathrm{~K}$, the 489 order-disorder transition $\alpha \rightarrow \alpha_{2}$ occurs, the order parameter of the $\alpha_{2} / \alpha$-phase 490 decreases, which results in the strain in the $\alpha_{2} / \alpha$-phase along the $c$-direction having a 491 dramatic decrease. In region IV, up to $1529 \mathrm{~K}$, the $\beta$-phase fraction increases sharply 492 (Figure 3), leading to a trend of an increase along the $c$-direction and a decrease in $a$ 493 direction in the $\alpha_{2} / \alpha$-phase, although questioned by large error bars. At higher 494 temperature, the anisotropic strain along the $a$-direction increases while that along the $495 \quad c$-direction decreases.

496 Figure 13 shows how temperature, aluminium content, order parameter and pressure 497 contribute to strain evolution in the $\gamma$-phase at a nominal pressure of $9.6 \mathrm{GPa}$ in the 498 different regions respectively. Because of this observed pressure loss, the anisotropic 499 part of strain, $(\Delta a / a-\Delta V / 3 V, \Delta c / c-\Delta V / 3 V)$ of the $\gamma$-phase is shown in Figure 14 . 500 The sample pressure is from $9.6 \mathrm{GPa}$ to $6.9 \mathrm{GPa}$ - a very similar order of magnitude 501 which would not considerably change the phase transformation behavior. Moreover, 502 this anisotropic part of lattice strain is subtracted by the pressure influence, but 503 relevant features with respect to order parameter can be extracted. The significant 
506 pressure of 9.6 $\mathrm{GPa}$ are discussed below:
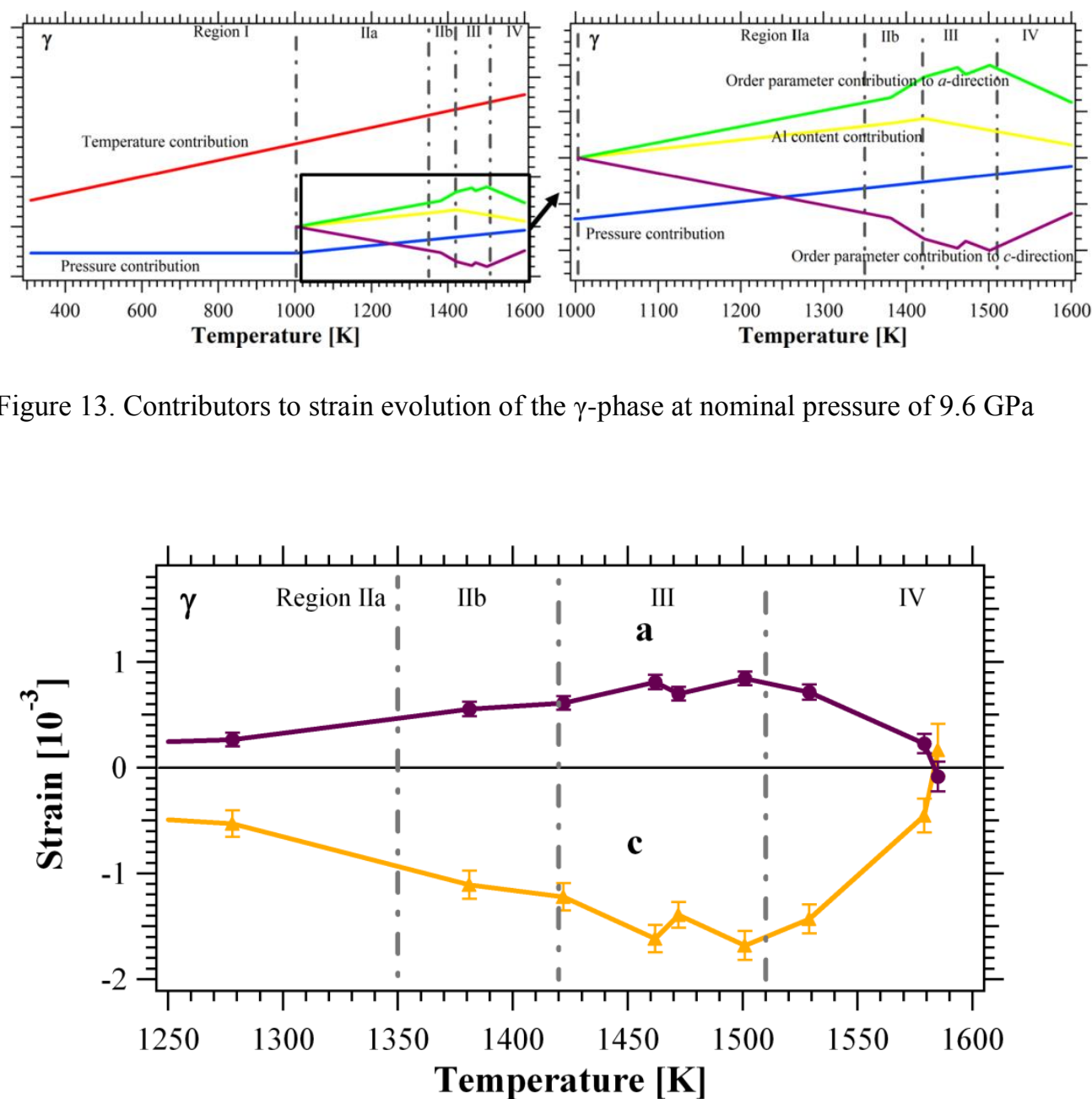

Figure 14. Dependence of the anisotropic lattice strain in the $\gamma$-phase along $a$ - (purple), $c$ - (brown) directions for Ti-45Al-7.5Nb-0.25C at $9.6 \mathrm{GPa}$

514 In region III, the transition of $\gamma \rightarrow \alpha \rightarrow \beta+\alpha$ [13] results in an increased aluminium

515 content in the $\gamma$-phase, therefore, the strain increase is lower compared to atmospheric

516 pressure. The kink at $1472 \mathrm{~K}$ is attributed to the order parameter increase as a result of

517 the fact that the $\gamma$-phase fraction decreases sharply in the temperature range 1462 to

$5181472 \mathrm{~K}$. In region $\mathrm{IV}$, the trend in strain evolution is almost the same as at 
519 atmospheric pressure, since the main contributing factor is the order parameter.

$520 \quad$ 4.3. Phase sequence at high pressure

521 Based on our experimental observations and the Rietveld analyses outlined above, a

522 new version of the sequence of phase changes occurring in the Ti-Al-7.5Nb-0.25C

523 alloy under a pressure of $9.6 \mathrm{GPa}$, is shown in Figure 15 in the region of the alloy

524 composition (delineated by the area between the two vertical dotted lines). This is the

525 first experimental evidence suggesting that during heating under high pressure the

526 sequence of phase development is $\alpha_{2}+\gamma, \alpha_{2}+\alpha+\gamma, \alpha_{2}+\alpha+\gamma+\beta, \alpha+\gamma+\beta, \alpha+\beta, L+\alpha+\beta, L+\beta$

527 and liquid L. Compared to the phase diagram suggested by Chladil et al. [19], it is

528 evident that the temperature range of the phase field $\alpha_{2}+\alpha+\gamma+\beta$ is extended under the

529 influence of a pressure of 9.6 GPa.

530

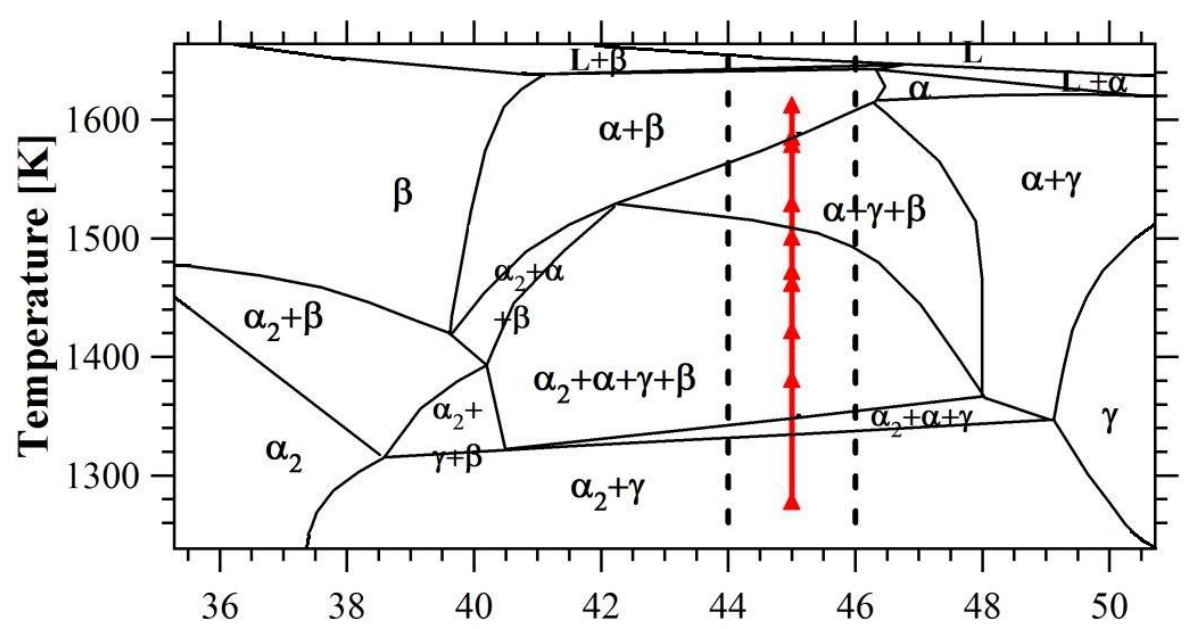

Mole fraction aluminium

Figure 15. Proposed sequence of phase changes upon heating a Ti-Al-7.5 Nb-0.25C alloy under a pressure of 9.6 GPa. The red triangles are based on the calculations of this study from the diffractions measured by Liss et al. [13], superimposed on a section of a schematic diagram of the phase compositions in the Ti-Al-7.5Nb-0.25C system. 
538 The quantitatively calculated contributions to strain evolution at standard atmospheric 539 pressure are listed in Table 1.

Table 1. Contribution to strain evolution at standard atmospheric pressure

\begin{tabular}{|c|c|c|c|c|}
\hline Phase & Strain direction & $\begin{array}{c}\text { Expansion } \\
\text { coefficient } \eta \\
{\left[10^{-6} \mathrm{~K}^{-1}\right]}\end{array}$ & $\begin{array}{c}\text { Increasing } \mathrm{Al} \\
\text { content }\left[10^{-6} \mathrm{~mol}^{-1}\right]\end{array}$ & $\begin{array}{c}\text { Atomic order } \\
\mathrm{S}(0 \rightarrow 1)\left[10^{-6}\right]\end{array}$ \\
\hline \multirow{2}{*}{$\alpha_{2} / \alpha$} & $a$ & $\Uparrow 12.075$ & $\Downarrow$ & $\Downarrow-695$ \\
\cline { 2 - 5 } & $c$ & $\Uparrow 12.047$ & $\Downarrow$ & $\Leftrightarrow 1390$ \\
\cline { 2 - 5 } & $V / 3$ & $\Uparrow 12.066$ & $\Downarrow-18959$ & $\Downarrow-4677^{*}$ \\
\hline \multirow{2}{*}{$\gamma$} & $a$ & $\Uparrow 12.412$ & $\Downarrow$ & $\Uparrow 9420^{*}$ \\
\cline { 2 - 5 } & $c$ & $\Uparrow 11.847$ & $\Downarrow$ & $\Leftrightarrow$ \\
\cline { 2 - 5 } & $V / 3$ & $\Uparrow 12.224$ & $\Downarrow-24079$ & $\Leftrightarrow$ \\
\hline
\end{tabular}

${ }^{*}$ Computed with lattice parameter values of Beaven [31]

544 The linear thermal expansion coefficients are $\eta_{\mathrm{a}}=12.075 \cdot 10^{-6} \mathrm{~K}^{-1}$ and $\eta_{\mathrm{c}}=$

$545 \quad 12.047 \cdot 10^{-6} \mathrm{~K}^{-1}$ for $\alpha_{2} / \alpha$-phase and $\eta_{\mathrm{a}}=12.412 \cdot 10^{-6} \mathrm{~K}^{-1}$ and $\eta_{\mathrm{c}}=11.847 \cdot 10^{-6} \mathrm{~K}^{-1}$ for $\gamma$ -

546 phase. They were extracted by fitting the curves of $a$ - and $c$-directions in region I of

547 Figures 4 and 5, since thermal expansion is proportional to the temperature change for 548 most solids, $\varepsilon_{\text {thermal }}=\eta \cdot \Delta T[28,32]$. In order to calculate the contribution of the 549 aluminium content of the $\alpha_{2} / \alpha$-phase, the slope of the strain (Figure 4 and Figure 5) 550 and the slope of the phase boundary lines (Figure 10(a)) in region IV were used since 551 the thermal expansion and the aluminium content both contribute to volumetric strain 552 evolution in this region. The contribution of aluminium to the lattice strains 
553 are $\partial \varepsilon /\left.\partial C\right|_{\alpha}=-18959 \cdot 10^{-6} \mathrm{~mol}^{-1}$ and $\partial \varepsilon /\left.\partial C\right|_{\gamma}=-24079 \cdot 10^{-6} \mathrm{~mol}^{-1}$ respectively, in good

554 agreement with the values reported by Dubrovinskaia et al. [33]. It should be noted 555 that the thermal expansion coefficient at high temperature will differ from the values 556 obtained by diffraction, as further factors may contribute, such as the creation of 557 lattice vacancies which do not change the lattice plane spacing but the length and 558 volume of a specimen. The order parameter contributions are $\partial \varepsilon / \partial S_{a}=-695 \cdot 10^{-6}$ and $559 \partial \varepsilon / \partial S_{c}=1390 \cdot 10^{-6}$ for $\alpha_{2} / \alpha$-phase along $a$ - and $c$ - directions respectively. The 560 experimental data points around the $\gamma$-solvus at $1476 \mathrm{~K}$, were chosen to calculate $561 \partial \varepsilon / \partial S$ for each component. In Figure 4, the steps across these data are $0.45 \cdot 10^{-3}$ and $5620.5 \cdot 10^{-3}$ for $a$ - and $c$ - directions respectively, which are recorded as $\Delta \varepsilon_{a}^{M}$ and $\Delta \varepsilon_{c}^{M}$ 563 below. Considering that $\Delta \varepsilon_{a}^{M}$ and $\Delta \varepsilon_{c}^{M}$ are not only influenced by the order parameter, 564 but also by volume expansion, these equations were modified as follows:

$$
\begin{gathered}
\Delta \varepsilon_{a}=\Delta \varepsilon_{a}^{M}-\Delta \varepsilon_{v}, \\
\Delta \varepsilon_{c}=\Delta \varepsilon_{a}^{M}-\Delta \varepsilon_{v}
\end{gathered}
$$

566 where $\Delta \varepsilon_{\mathrm{v}}$ represents the slope of the average expansion, i.e. $1 / 3$ of the volume 567 expansion curve. From Figure A.3, the slope of the order parameter $(\Delta S)$ across this 568 step is 0.456 , resulting in $\partial \varepsilon / \partial S_{a}=\Delta \varepsilon_{\mathrm{a}} / \Delta \mathrm{S}=-695 \cdot 10^{-6}$ and $\partial \varepsilon / \partial S_{c}=\Delta \varepsilon_{\mathrm{c}} / \Delta \mathrm{S}=$ $5691390 \cdot 10^{-6}$. The order parameter of the $\gamma$ phase was estimated in a different way, since 570 no order parameter nor site occupation have been evaluated by Yeoh et al. [21]. The 571 fully disordered $\gamma$-phase would be $f c c$ with a $c / a$ axis ratio of one, and the atomic 572 volume is conserved during the order-disorder phase change. The lattice parameters of 573 the stoichiometric alloy have been reported by Beaven and Pfullman [31] and 574 extracted as $a_{50}=4.0176 \AA$ and $c_{50}=4.0745 \AA$, for the fully ordered crystal structure. 


$$
a_{f c c}=\sqrt[3]{a_{50}^{2} \times c_{50}}
$$

575 leading to $a_{f c c}=4.03648 \AA$. When the crystal structure changes from a disordered

$576(\mathrm{~S}=0)$ to a fully ordered structure $(\mathrm{S}=1)$, the influence of the structure parameter on

577 strain development is calculated as $\partial \varepsilon / \partial S_{a}=\left(a_{50^{-}}-a_{f c c}\right) / a_{f c c}=-4677 \cdot 10^{-6}$ and

$578 \quad \partial \varepsilon / \partial S_{c}=\left(c_{50}-a_{f c c}\right) / a_{f c c}=9420 \cdot 10^{-6}$, respectively.

579 Table 2 lists quantitative contributions to the strain evolutions at high pressure. The

580 thermal expansion coefficients are calculated by linear curve fitting based on the

581 strains in region I (Figures 6 and 7). They are $\eta_{\mathrm{a}}=8.126 \cdot 10^{-6} \mathrm{~K}^{-1}, \eta_{\mathrm{c}}=9.030 \cdot 10^{-6} \mathrm{~K}^{-1}$

582 for the $\alpha_{2} / \alpha$-phase and $\eta_{\mathrm{a}}=8.371 \cdot 10^{-6} \mathrm{~K}^{-1}, \eta_{\mathrm{c}}=8.359 \cdot 10^{-6} \mathrm{~K}^{-1}$ for $\gamma$-phase. The

583 expansion coefficients are approximately $8 \times 10^{-6} \mathrm{~K}^{-1}$ under a compressive pressure of

$5849.6 \mathrm{GPa}$ whereas it is $12 \times 10^{-6} \mathrm{~K}^{-1}$ under atmospheric pressure. Hence, a compressive

585 pressure of $9.6 \mathrm{GPa}$ decreases the expansion coefficient to $67 \%$ of the value at

586 atmospheric pressure. An increase of aluminium content in the respective phases

587 would lead to a decrease in the strain along the $a$ - and $c$-directions, as well as a

588 decrease in the volumetric strain. The application of an applied compressive pressure

589 caused the strain to decrease in both phases. Liss et al. [13] has already calculated the

590 strain decrease in the $\alpha_{2} / \alpha$-phase per $1 \mathrm{GPa}$ is $2266 \cdot 10^{-6}$ along $a$-direction and

$5912189 \cdot 10^{-6}$ along the $c$-direction from the same experimental data. The corresponding

592 strain decrease in the $\gamma$-phase is $2206 \cdot 10^{-6}$ along $a$-direction and $2293 \cdot 10^{-6}$ in the $c$ -

593 direction [13].

594

595

Table 2. Contributions to strain evolution at high pressure

\begin{tabular}{|l|l|l|l|l|}
\hline Phase & Strain direction & Expansion coefficient & Increasing Al & Pressure $^{*}$ \\
\hline
\end{tabular}




\begin{tabular}{|c|c|c|c|c|}
\hline & & $\eta\left[10^{-6} \mathrm{~K}^{-1}\right]$ & content & {$\left[10^{-6} \mathrm{GPa}^{-1}\right]$} \\
\hline \multirow{3}{*}{$\alpha_{2} / \alpha$} & $a$ & $\Uparrow 8.126$ & $\Downarrow$ & $\Downarrow 2266$ \\
\hline & $c$ & 介 9.030 & $\Downarrow$ & $\Downarrow 2189$ \\
\hline & $V$ & $\Uparrow 8.427 \times 3$ & $\Downarrow$ & $\Downarrow 2240 \times 3$ \\
\hline \multirow{3}{*}{$\gamma$} & $a$ & $\Uparrow 8.371$ & $\Downarrow$ & $\Downarrow 2206$ \\
\hline & $c$ & $\Uparrow 8.335$ & $\Downarrow$ & $\Downarrow 2293$ \\
\hline & $V$ & $\Uparrow 8.359 \times 3$ & $\Downarrow$ & $\Downarrow 2235 \times 3$ \\
\hline
\end{tabular}




\section{CONCLUSIONS}

599 Lattice strain evolution in a Ti-45Al-7.5Nb-0.5C and a Ti-45Al-7.5Nb-0.25C alloy

600 respectively was determined by in-situ experiments using high-energy X-rays at

601 synchrotron storage rings. The temperature dependence of lattice strain evolution of

602 the Ti-45Al-7.5Nb-0.5C alloy was studied at atmospheric pressure while the lattice

603 strain evolution in a Ti-45Al-7.5Nb-0.25C was determined as a function of

604 temperature under a compressive pressure of 9.6 GPa.

605 - Lattice strain evolution is determined by thermal expansion, changes in the 606 aluminium content of the respective phases and the extent to which the respective

607 phases are ordered under atmospheric pressure. Their interaction and respective 608 quantitative values to lattice strain changes have been obtained, which provides 609 valuable data to predict strain evolution in the future.

610 - Pressure has a determining influence on strain evolution in the alloys. A 611 consequence of this finding is that the magnitude of the lattice strain can be 612 manipulated by these four factors during manufacture. Hence, inter-granular 613 stresses can be reliably predicted, minimized and controlled in order to manipulate 614 the mechanical properties of candidate titanium aluminide alloys.

615 - The application of high pressure increases the eutectoid temperature $T_{\text {eu }}$ and the 616 temperature at which the transformation of the $\gamma$-phase is completed, $T_{\gamma, \text { solv }}$. The 617 linear thermal expansion coefficient of the alloy investigated is about $1 / 3\left(4 \times 10^{-6}\right.$ $618 \mathrm{~K}^{-1}$ ) lower under a pressure of $9.6 \mathrm{GPa}$, than under standard atmospheric pressure. 
619 - Based on the experimental observations, a new version of the sequence of phase 620 changes occurring at high pressure is proposed and illustrated with reference to a

621 schematic phase diagram. This portion of the phase diagram shows that under 622 high pressure, the $\alpha_{2}+\alpha+\gamma+\beta$ phase field is stabilized over a wide range of temper623 ature.

624 - Lattice strains can be used to indicate the occurrence of phase transformations and 625 changes in composition, which are otherwise difficult to determine.

626 - The $c / a$ ratio of both the $\alpha_{2} / \alpha$ - and $\gamma$-phases provides valuable insight into the ex627 tent to which these phases are ordered (as assessed by the order parameter).

628 - A discontinuity in the $c / a$ ratio is an indication of the order-disorder transition $629 \quad \alpha_{2} \rightarrow \alpha$

630 The present findings are of generic importance with respect to lattice parameter 631 evaluation and are relevant to a multitude of intermetallic systems.

\section{$632 \quad$ 6. Acknowledgments}

633 The authors are grateful for the financial contributions made by the China Scholarship

634 Council (CSC), The University of Wollongong and the Australian Nuclear Science 635 and Technology Organization (ANSTO). The provision of and access to experimental 636 equipment at beamline BL04B1, SPring-8 (under proposal number 2013B1157) and 637 the availability of data from experiment MA-77 taken at ID15A, ESRF and their 638 experimentalists [21] are gratefully recognized. Dr. habil. Arno Bartels, Technische 639 Universität Hamburg-Harburg, Germany, is acknowledged for providing the alloys 640 used in this study. 
642 7. Appendix

643 The concept of an order-parameter was introduced by Bragg and William [34]. They

644 defined an order parameter $\mathrm{S}$ as follows:

645

$$
\mathrm{S}=\frac{p-r}{1-r}
$$

646 where $p$ is the probability that an atom resides in an ordered position $r$. For example,

647 if an aluminium atom remains in its ordered position (the red sites depicted in Figure

648 A.1) and no other atoms occupy these positions, the value of $p$ is $1 . r$ is the fraction of

649 aluminium atoms residing in a fully ordered lattice. The structure of a fully ordered

650 lattice $\alpha_{2}-\mathrm{Ti}_{3} \mathrm{Al}$ is shown in Figure A.1. Aluminium atoms occupy $25 \%$ of the lattice

651 positions, which means that $r$ has a value of 0.25 .

652 The order-disorder transition refers to the case where aluminium and titanium atoms

653 in the crystal lattice randomly interchange anti-sites. For example, titanium atoms

654 have the possibility of residing in red positions.

655
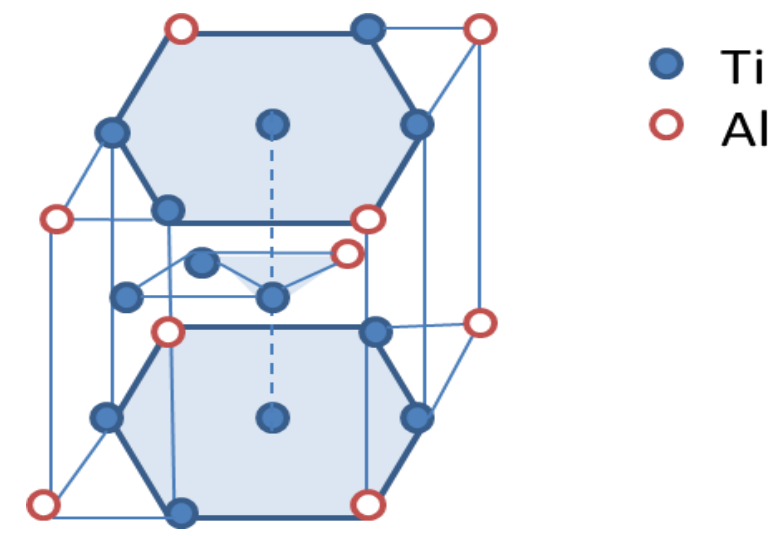

O Al

Figure A. 1 the structure of ordered lattice $\alpha_{2}$ phase $\left(\mathrm{Ti}_{3} \mathrm{Al}\right)$

658

659 Figure A.2 shows the order parameter (S) for the $\alpha_{2} / \alpha$-phase, calculated from the site 
660 occupancies reported by Yeoh et al. [21] (without taking into account the change in

661 aluminium concentration). The sharp decrease in order parameter coincides with the

662 sudden change in the lattice strain shown in Figure 5. This observation indicates that a

663 higher $c / a$ ratio reflects a more ordered structure. It is instructive to compare the

664 occurrence of this sudden change in order parameter to changes in the $c / a$-ratio of the

$665 \alpha_{2} / \alpha$-phase as a function of temperature, as shown in Figure A.3. The order parameter

666 changes in the same fashion as the $c / a$ ratio in the $\alpha_{2} / \alpha$-phase and hence, it seems

667 reasonable to use the $c / a$ ratio as a measure of the degree to which the $\alpha_{2} / \alpha$-phase is

668 ordered.

669

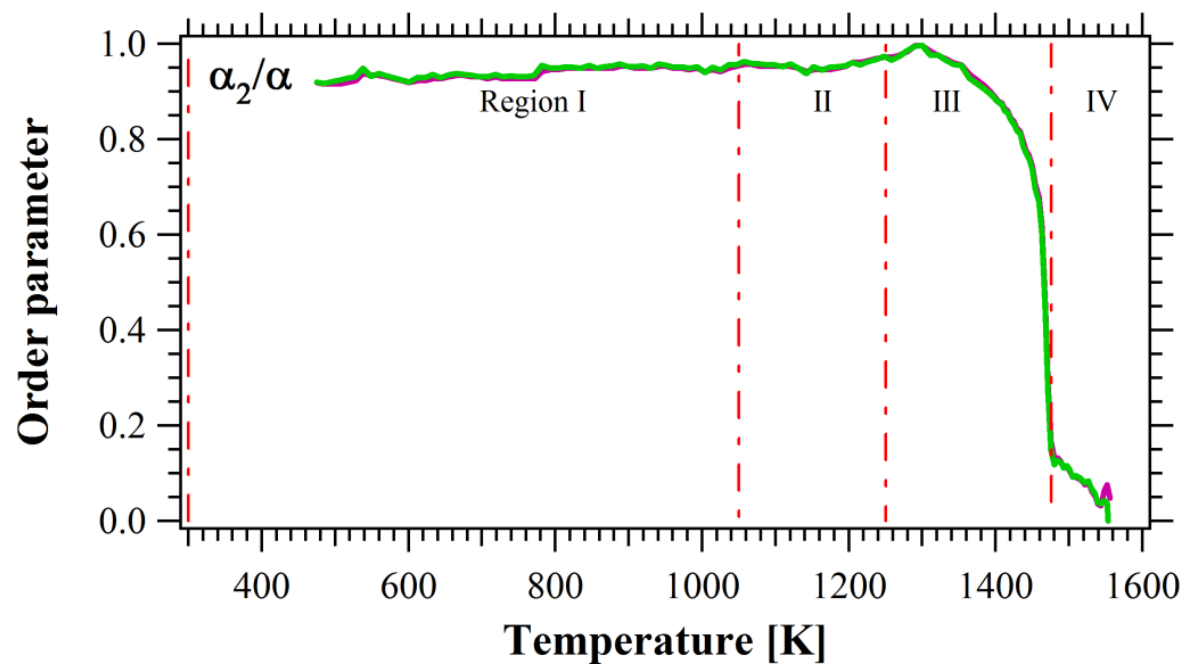




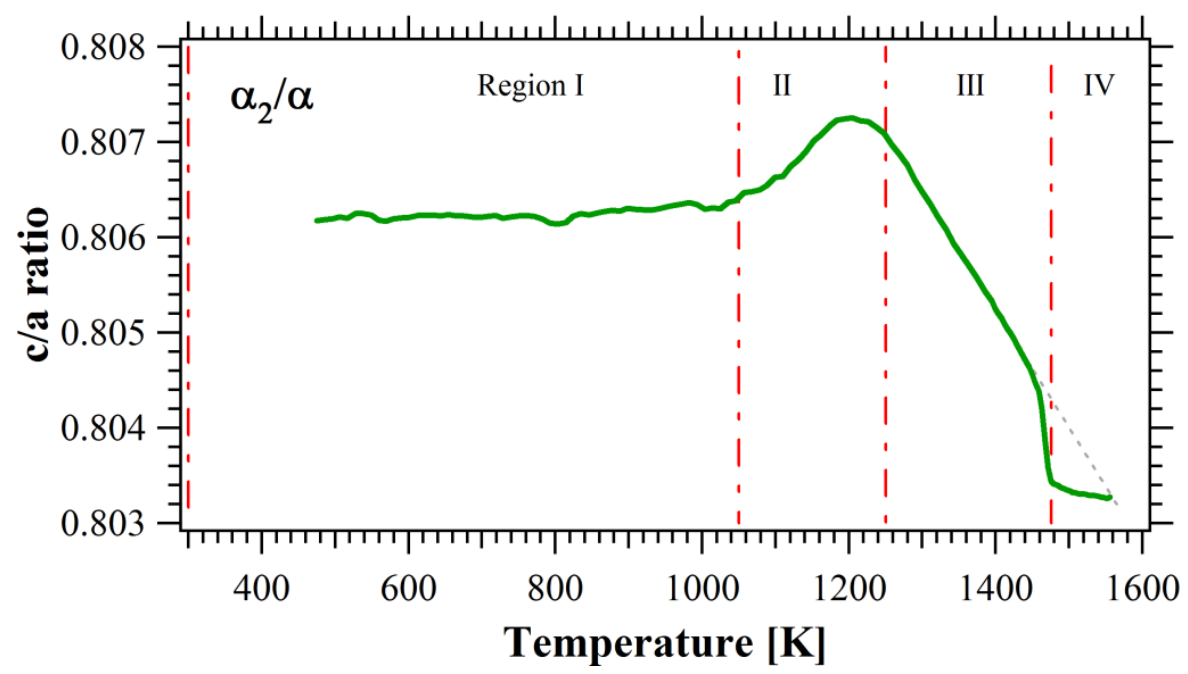

Figure A.3. The $c / a$ ratio of the $\alpha_{2} / \alpha$-phase for a Ti-45Al-7.5Nb-0.5C alloy at atmospheric pressure [21].

676 
[1] R. Yang, "Advances and challenges of TiAl base alloys," Acta Metallurgica Sinica, vol. 51, 2015.

[2] K. H. Matucha, "Materials Science and Technology: a Comprehensive Treatment. Vol. 8. Structure and Properties of Nonferrous Alloys," $V C H$ Verlagsgesellschaft mbH, P. O. Box 1011 61, Weinheim, D-69451, Germany, 1996. 837, 1996.

[3] Y.-W. Kim, "Ordered intermetallic alloys, part III: gamma titanium aluminides," Jom, vol. 46, pp. 30-39, 1994.

[4] H. F. Chladil, H. Clemens, G. A. Zickler, M. Takeyama, E. Kozeschnik, A. Bartels, et al., "Experimental studies and thermodynamic simulation of phase transformations in high $\mathrm{Nb}$ containing $\gamma$-TiAl based alloys," International Journal of Materials Research, vol. 98, pp. 1131-1137, 2007.

[5] FRANCE-METALLURGIE. (2014). 80,000 tons World's Largest Closed Die Forging Press help to develop China Jet Fighter J-11 (US). Available: http://www.france-metallurgie.com/china-80000-tons-world-s-largest-closeddie-forging-presses-help-to-develop-the-f-11-us/

[6] FRANCE-METALLURGIE. (2016). OTTO FUCHS orders 54,000 Tons Closed Die Forging Press from SMS (US). Available: http://www.francemetallurgie.com/otto-fuchs-orders-54000-tons-closed-die-forging-press-from$\underline{\text { sms-us/ }}$

[7] F. Appel, J. D. H. Paul, and M. Oehring, Gamma titanium aluminide alloys: science and technology: John Wiley \& Sons, 2011.

[8] K. Khan, C. Sanchez, M. Thomas, S. Naka, and -. C. Office National d'Etudes et de Recherches Aerospatiales "Development of third generation castable gamma titanium aluminides: role of solidification paths," 1997.

[9] Y. Jin, J. N. Wang, J. Yang, and Y. Wang, "Microstructure refinement of cast TiAl alloys by $\beta$ solidification," Scripta Materialia, vol. 51, pp. 113-117, 7// 2004.

[10] K.-D. Liss, T. Schmoelzer, K. Yan, M. Reid, M. Peel, R. Dippenaar, et al., "In situ study of dynamic recrystallization and hot deformation behavior of a 
multiphase titanium aluminide alloy," Journal of Applied Physics, vol. 106, p. 113526, 2009.

[11] H. Clemens and S. Mayer, "Design, processing, microstructure, properties, and applications of advanced intermetallic TiAl alloys," Advanced Engineering Materials, vol. 15, pp. 191-215, 2013.

[12] H. Clemens, M. Schloffer, E. Schwaighofer, R. Werner, A. Gaitzenauer, B. Rashkova, et al., "Advanced $\beta$-Solidifying Titanium AluminidesDevelopment Status and Perspectives," in MRS Proceedings, 2013, pp. 3-16.

[13] K.-D. Liss, K.-I. Funakoshi, R. Dippenaar, Y. Higo, A. Shiro, M. Reid, et al., "Hydrostatic Compression Behavior and High-Pressure Stabilized $\beta$-Phase in $\gamma$-Based Titanium Aluminide Intermetallics," Metals, vol. 6, p. 165, 2016.

[14] K.-D. L. Tapan Chatterji, and Thomas Tschentscher, "Anomalous Thermal Expansion Due to Magnetism in EuAs3 and MnS2," ESRF Highlights, 1997.

[15] T. Chatterji, K. Liß, G. McIntyre, M. Weiden, R. Hauptmann, and C. Geibel, "The ground state of NaV2O5," Solid state communications, vol. 108, pp. 2326, 1998.

[16] K.-D. Liss, A. Bartels, A. Schreyer, and H. Clemens, "High-energy X-rays: a tool for advanced bulk investigations in materials science and physics," Textures and Microstructures, vol. 35, pp. 219-252, 2003.

[17] K. Yan, D. G. Carr, S. Kabra, M. Reid, A. Studer, R. P. Harrison, et al., "In Situ Characterization of Lattice Structure Evolution during Phase Transformation of $\mathrm{Zr}-2.5 \mathrm{Nb}, "$ Advanced Engineering Materials, vol. 13, pp. 882-886, 2011.

[18] J. C. Schuster and M. Palm, "Reassessment of the binary aluminum-titanium phase diagram," Journal of phase equilibria and diffusion, vol. 27, pp. 255$277,2006$.

[19] H. F. Chladil, H. Clemens, H. Leitner, A. Bartels, R. Gerling, and W. T. Marketz, "Experimental Studies of Phase Transformations in a Carbon Containing $\mathrm{Ti}-45 \mathrm{Al}-7.5 \mathrm{Nb}$ Alloy and Related Thermodynamic Simulations," Advanced Engineering Materials, vol. 7, pp. 1131-1134, 2005.

[20] R. D. Shull and J. P. Cline, "High temperature X-ray diffractometry of Ti-Al 
alloys," in Materials Chemistry at High Temperatures, ed: Springer, 1990, pp. 95-117.

[21] L. A. Yeoh, K.-D. Liss, A. Bartels, H. Chladil, M. Avdeev, H. Clemens, et al., "In situ high-energy X-ray diffraction study and quantitative phase analysis in the $\alpha+\gamma$ phase field of titanium aluminides," Scripta Materialia, vol. 57, pp. 1145-1148, 2007.

[22] L. Lutterotti, "Total pattern fitting for the combined size-strain-stress-texture determination in thin film diffraction," Nuclear Instruments and Methods in Physics Research Section B: Beam Interactions with Materials and Atoms, vol. 268, pp. 334-340, 2010.

[23] L. Lutterotti, S. Matthies, and H. Wenk, "MAUD (Material Analysis Using Diffraction): a user friendly Java program for Rietveld texture analysis and more," in Proceeding of the twelfth international conference on textures of materials (ICOTOM-12), 1999, p. 1599.

[24] H. Chladil, H. Clemens, H. Leitner, A. Bartels, R. Gerling, F.-P. Schimansky, et al., "Phase transformations in high niobium and carbon containing $\gamma$-TiAl based alloys," Intermetallics, vol. 14, pp. 1194-1198, 2006.

[25] Y. T. a. Y. Higo, "BL04B1: In situ observation of high pressure phase change of simple material," Spring-8, Kouto, Kyogo, Janpan, Tutorial 4Sep. 2007

[26] T. Katsura, K.-i. Funakoshi, A. Kubo, N. Nishiyama, Y. Tange, Y.-i. Sueda, et al., "A large-volume high-pressure and high-temperature apparatus for in situ X-ray observation, 'SPEED-Mk. II'," Physics of the Earth and Planetary Interiors, vol. 143, pp. 497-506, 2004.

[27] R. Gerling, H. Clemens, and F. P. Schimansky, "Powder Metallurgical Processing of Intermetallic Gamma Titanium Aluminides," Advanced Engineering Materials, vol. 6, pp. 23-38, 2004.

[28] F. Abe, T.-U. Kern, and R. Viswanathan, Creep-resistant steels: Elsevier, 2008.

[29] V. Witusiewicz, A. Bondar, U. Hecht, and T. Y. Velikanova, "The Al-B-Nb-Ti system: IV. Experimental study and thermodynamic re-evaluation of the binary $\mathrm{Al}-\mathrm{Nb}$ and ternary $\mathrm{Al}-\mathrm{Nb}-\mathrm{Ti}$ systems," Journal of Alloys and Compounds, vol. 472, pp. 133-161, 2009. 
[30] M. Asta, D. de Fontaine, and M. Van Schilfgaarde, "First-principles study of phase stability of Ti-Al intermetallic compounds," Journal of materials research, vol. 8, pp. 2554-2568, 1993.

[31] P. Beaven and T. Pfullmann, "Stoichiometry effects in TiAl and TiAlCr alloys," Journal of materials science \& technology, vol. 10, pp. 321-330, 1994.

[32] O. A. Bauchau and J. I. Craig, Structural analysis: with applications to aerospace structures vol. 163: Springer Science \& Business Media, 2009.

[33] N. Dubrovinskaia, M. Vennström, I. Abrikosov, R. Ahuja, P. Ravindran, Y. Andersson, et al., "Absence of a pressure-induced structural phase transition in Ti 3 Al up to 25 GPa," Physical Review B, vol. 63, p. 024106, 2000.

[34] W. L. Bragg and E. J. Williams, "The effect of thermal agitation on atomic arrangement in alloys," Proceedings of the Royal Society of London. Series A, Containing Papers of a Mathematical and Physical Character, vol. 145, pp. 699-730, 1934. 